\title{
Factorisations for Partition Functions of Random Hermitian Matrix Models
}

\author{
D.M. Jackson ${ }^{1}$, M.J. Perry ${ }^{2}$, T.I. Visentin ${ }^{3}$ \\ ${ }^{1}$ Department of Combinatorics and Optimization, University of Waterloo, Waterloo, Ontario, \\ Canada. E-mail: dmjackson@dragon.uwaterloo.ca \\ ${ }^{2}$ Department of Applied Mathematics and Theoretical Physics, Cambridge University, Silver Street, \\ Cambridge CB3 9EW, England \\ ${ }^{3}$ Department of Mathematics, University of Winnipeg, Winnipeg, Manitoba, Canada
}

Received: 8 April 1994 / Accepted: 23 October 1995

\begin{abstract}
The partition function $Z_{N}$, for Hermitian-complex matrix models can be expressed as an explicit integral over $\mathbb{R}^{N}$, where $N$ is a positive integer. Such an integral also occurs in connexion with random surfaces and models of two dimensional quantum gravity. We show that $Z_{N}$ can be expressed as the product of two partition functions, evaluated at translated arguments, for another model, giving an explicit connexion between the two models. We also give an alternative computation of the partition function for the $\phi^{4}$-model. The approach is an algebraic one and holds for the functions regarded as formal power series in the appropriate ring.
\end{abstract}

\section{Contents}

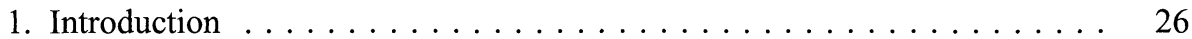

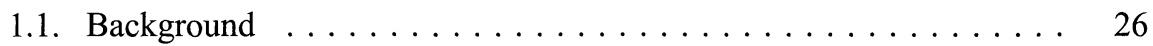

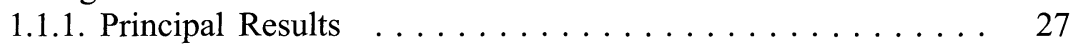

1.1.2. Organisation of the Paper ................... 27

1.2. Matrix Models, the $\phi^{4}$-Model and the Penner Model . . . . . . 28

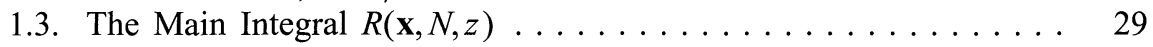

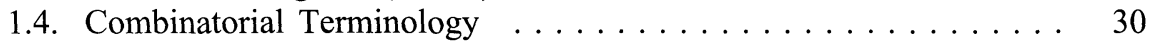

1.4.1. Graphs ....................... 30

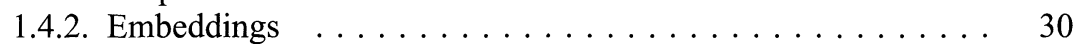

1.4.3. The Genus Series . . . . . . . . . . . . . 30

1.5. The Approach ..................... 30

1.6. The Main Theorems ...................... 31

1.6.1. Factorisation of the Partition Function for Matrix Models . . 31

1.6.2. Combinatorial Maps . . . . . . . . . . . . . 31

1.6.3. Maps and Vertex 4-Regular Maps ............ 32

1.6.4. A Relationship between the $\phi^{4}$ - and Penner-Models .... 32

1.6.5. A Reformulation for the $\phi^{4}$-Model . . . . . . . . . . 33

1.6.6. A Factorisation for Hypermaps . . . . . . . . . . . 33

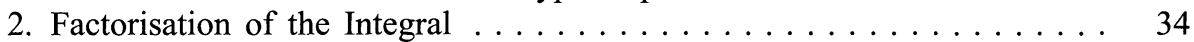

2.1. The Character Formulation of the Integral . . . . . . . . . . . 34 
2.2. Factorisation of Characters $\ldots \ldots \ldots \ldots \ldots \ldots \ldots \ldots \ldots$

2.3. The Factorisation of $R_{4}(x, y, z) \ldots \ldots \ldots \ldots \ldots \ldots \ldots \ldots$

2.4. Permutations and $R_{\mathcal{N}}(x, y, z) \ldots \ldots \ldots \ldots \ldots \ldots \ldots \ldots \ldots \ldots \ldots$

3. The $\phi^{4}$ - and Penner-Models $\ldots \ldots \ldots \ldots \ldots \ldots \ldots \ldots \ldots$

3.1. Enumerative Information $\ldots \ldots \ldots \ldots \ldots \ldots \ldots \ldots 40$

3.1.1. Combinatorial Operations on Sets ........... 41

3.1.2. The Inverse Problem and Refinement . . . . . . . . 42

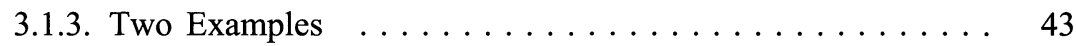

3.2. Rotation Systems $\ldots \ldots \ldots \ldots \ldots \ldots \ldots \ldots \ldots \ldots \ldots \ldots$

3.3. A Combinatorial Construction for Feynman Maps ......... 47

3.3.1. Suppression of Monovalent Vertices $\ldots \ldots \ldots \ldots \ldots 47$

3.3.2. Suppression of Bivalent Vertices ............ 48

4. Reformulation of the Partition Function for the $\phi^{4}$-Model . . . . . . . 49

4.1. Specialisation of Trees and 1-Vertex Maps $\ldots \ldots \ldots \ldots \ldots 50$

4.2. The Genus Series for Regular Maps $\ldots \ldots \ldots \ldots \ldots \ldots \ldots .51$

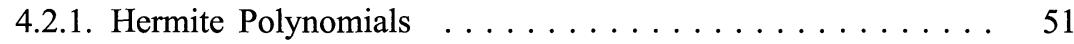

4.2.2. An Inverse Problem and Superposition of Matchings .... . 52

4.2.3. The Genus Series for Regular Maps $\ldots \ldots \ldots \ldots \ldots \ldots 52$

4.3. Basis Polynomials for the Genus Series $\ldots \ldots \ldots \ldots \ldots \ldots$. 54

4.3.1. Parity-Respecting Forms $\ldots \ldots \ldots \ldots \ldots \ldots \ldots \ldots$

4.3.2. Degree-Respecting Forms $\ldots \ldots \ldots \ldots \ldots \ldots \ldots . \ldots 5$

4.3.3. Topologically Good Forms $\ldots \ldots \ldots \ldots \ldots \ldots \ldots 5$

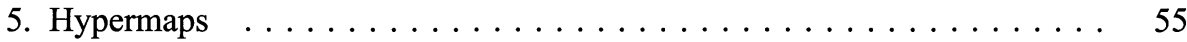

5.1. The Embedding Theorem for Hypermaps $\ldots \ldots \ldots \ldots \ldots \ldots 55$

5.2. The Factorisation $\ldots \ldots \ldots \ldots \ldots \ldots \ldots \ldots \ldots \ldots \ldots$

\section{Introduction}

In this section we describe briefly the background to matrix models and give the statements of the two principal results, together with the supporting results that are also of interest. Background material on graph embeddings is also provided, with the notational apparatus for symmetric functions.

1.1. Background. The partition function is defined explicitly by

$$
Z_{N}\left(u_{2}, u_{3}, \ldots ; \beta\right)=\int_{\mathscr{V}_{N}} e^{-\beta \operatorname{trace} U(\mathbf{M})} d \mathbf{M},
$$

where $\mathbf{M} \in \mathscr{V}_{N}$, the set of $N \times N$ Hermitian complex matrices, $U(\xi)=u_{2} \xi^{2}+$ $u_{3} \xi^{3}+\cdots$, and the weights $u_{2}, u_{3}, \ldots$ are real.

In a seminal paper ' $t$ Hooft [18] studied an approach to quantum chromodynamics with $N$ species of quarks that paved the way to a partial understanding of quark confinement. He showed, by using physical arguments, that the partition function for the theory was, in a particular limit, given by counting the number of ribbon graphs (or fat graphs). These come about by supposing that quark-antiquark pairs are permanently attached to each other, so that the pair sweeps out a two-dimensional surface in space-time. The two edges of this surface are the quark and antiquark, and the surface itself is imagined to be the result of the complicated interaction of the gluons. In some sense, the surface can be regarded as a meson, and so gives a 
physical picture as to why hadronic string theory should have some form of success. In his model, interactions of mesons come about where these two-dimensional surfaces interact. Suppose that $k$ such surfaces meet to form a $k$-point vertex with coupling constant $u_{k} k$ !, then he showed that the resultant partition function was given by simply summing all possible graphs weighted by the appropriate coupling constants. Subsequently, it was shown by Bessis, Itzykson and Zuber [3] that the generating series for this combinatorial model was given precisely by the $N \times N$ Hermitian matrix model devised somewhat earlier by Wigner [33] and Dyson [9].

1.1.1. Principal Results. This paper gives a self-contained account of some new and surprising relations between $Z_{N}$ found in different matrix models. The principal results are Theorem $\mathrm{A}$ and Theorem $\mathrm{E}$. The first result, Theorem A, relates the partition function for the Penner model [28] to that of the $\phi^{4}$-model. The factorisation is stated conveniently in terms of

$$
\mathscr{Z}_{N}(U, \beta)=\int_{\mathscr{V}_{N}} e^{-\frac{1}{2} \operatorname{trace} \mathbf{M}^{2}+\beta \operatorname{trace} U(\mathbf{M})} d \mathbf{M} / \int_{\mathscr{V}_{N}} e^{-\frac{1}{2} \operatorname{trace} \mathbf{M}^{2}} d \mathbf{M},
$$

where $U(\xi)=\sum_{k \geqq 1} \frac{1}{k} \sqrt{z}^{k} \xi^{k}$. It states that

$$
\mathscr{Z}_{2 N}\left(U_{4}, 1\right)=\mathscr{Z}_{N}\left(\widehat{U}, N+\frac{1}{2}\right) \cdot \mathscr{Z}_{N}\left(\widehat{U}, N-\frac{1}{2}\right),
$$

where $U_{4}(\xi)=\frac{1}{4} z^{2} \xi^{4}$ and $\widehat{U}(\xi)=\log (1-\sqrt{z} \xi)^{-1}$. The proof is an algebraic and combinatorial one. No analytic proof is available.

This result shows that the partition function for the $\phi^{4}$-model is completely determined by the partition function for the Penner model. Note that this result determines $\mathscr{Z}_{2 N}\left(U_{4}, 1\right)$ for an arbitrary value of the coupling constant $z$. Although we have found the $\phi^{4}$-partition function only for even-dimensional matrices, it is plausible that the result is generally true because $\mathscr{Z}_{2 N}\left(U_{4}, 1\right)$ has coefficients, as a series in $z$, that are polynomial in $N$.

Theorem $\mathrm{E}$ moves the computation of the partition function for the $\phi^{4}$-model to a new context, namely the determination of the constant term in a multivariate formal Laurent series.

1.1.2. Organisation of the Paper. Section 1 contains a brief account of background material on matrix models and 2-d quantum gravity, embeddings, maps and the generating series associated with them. Statements of the six main theorems and their purpose are also given. A map is a ribbon graph re-interpreted as a graph proper embedding in a two-dimensional surface.

In Sect. 2, the theory of symmetric functions is used, without reference to maps, to express the integral representation of $Z_{N}(U)$ as a character sum (9). A factorisation is given for characters of irreducible representations of the symmetric group at certain group elements, and this is then used to complete the factorisation (Theorem A) of $Z_{N}(U)$. The factorisation for characters is also used to obtain a comparable result for hypermaps in Sect. 5, which is related to the two-matrix model.

The remaining sections make extensive use of maps, the terminology of which is explained during the course of the paper. 
Section 3 contains a brief account of the combinatorial operations that are needed for this study of maps.

- A connexion (Theorem B) between $Z_{N}(U)$ and the generating series for the number of distinct maps embedded in a surface of prescribed genus, that is, the genus series, is then established.

- A relationship (Theorem C) is obtained between the genus series, $M_{4}$, for maps with vertices of degree four alone, and the genus series, $M_{\mathcal{N}}$, for all maps.

- Combinatorial constructions are used for suppressing the occurrence of monovalent (of degree one) and bivalent (of degree two) vertices in embeddings of graphs. This leads to the relationship (Theorem D) between $M_{4}$ (the $\phi^{4}$-model, vide inf.) and the genus series, $M_{\overline{1}}$, for maps with no vertices of degrees one or two (the Penner model, vide inf.). We refer to maps with no monovalent or bivalent vertices as Feynman maps. This result provides us with another way of getting new information about $\phi^{4}$-theory from the types of matrix models.

Section 4 gives a formulation (Theorem $\mathrm{E}$ ) for the genus series for vertex-regular maps. A vertex-regular map is a map, all of whose vertices have the same degree, so a $k$-regular map has only vertices of degree $k$. This then gives a reformulation of the problem for determining $M_{4}$.

Section 5 extends the theory to 2-face-colourable maps, a class of maps that may be associated with the 2-matrix model, to obtain a relationship (Theorem F) between the genus series for a class of face 2-coloured maps (hypermaps). This question is not explored further in this paper.

1.2. Matrix Models, the $\phi^{4}$-Model and the Penner Model. Random surfaces occur in the study of matter coupled to 2-dimensional gravity where variation in geometry is an essential feature. The latter furnishes a toy model for string theory $[4,8,12$, 13]. It is well known that $\log Z_{N}(U)$ has an interpretation as the sum over all two dimensional surfaces of genus $g$. For Hermitian-complex models, the case that is considered here, the surfaces are orientable and $\log Z_{N}(U)$ corresponds to a combinatorial sum over all connected graphs $G$ embedded in such surfaces. The graphs can be regarded as Feynman graphs. A more general case is the real symmetric matrix model in which the corresponding surfaces are locally orientable (the union of the orientable and non-orientable cases). We will not consider these here.

If such a graph has $v(G)$ vertices, $e(G)$ edges (links) and $f(G)$ faces then $2-2 g(G)=v(G)-e(G)+f(G)$, by the Euler-Poincaré Theorem. In physics terminology, $f(G)$ is the number of loops in a Feynman graph. However, the combinatorial usage is somewhat different, and this latter usage will be maintained in the remainder of the paper. To make contact with string theory, the double scaling limit is taken, which amounts to sending $N$ to infinity at the same time as the "area" of a surface is kept constant. The definition of area that is needed for this purpose is $a(G)=\frac{1}{2} \sum_{v}\left(d_{G}(v)-2\right)$, where $d_{G}(v)$ is the degree of the vertex $v$ in $G$ (the number of edges in $G$ incident with $v$ ) and the sum is over the vertex-set of $G$.

Under rescaling of the quadratic term $\frac{1}{2} \mathbf{M}^{2}$ in $\beta U(\mathbf{M})$ to $\frac{1}{2} N \mathbf{M}^{2}$, the weights $u_{j}$ transform to $u_{j} N \sqrt{N / \beta}^{j-2}$, for $j \geqq 3$. The expansion of $\log Z_{N}(U)$ in powers of $N^{-1}$ then has the form [12]

$$
\log Z_{N}(U)=t+\sum_{G} N^{2(1-g)}\left(\frac{N}{\beta}\right)^{a} w_{U}(G), \quad \text { where } w_{U}(G)=\prod_{j \geqq 3} \frac{u_{j}^{m_{j}(G)}}{j^{m_{j}(G)} m_{j}(G) !},
$$


and where also $t$ has emerged as a collection of ignorable terms of the form $c_{0}+$ $c_{1} \log \beta$, and $m_{j}(G)$ is the number of vertices of degree $j$ in $G$. The sum of Feynman graphs, generated by a suitable $U$, restricted to genus $g$ and areas $a$, is a discrete approximation to the sum over random surfaces of genus $g$ and area $a$, and it is believed that this should reduce to the continuum definition in the double scaling. Since $j \geqq 3$, vertices of degree one and two are excluded from the sum. The genus is invariant under deletion of vertices of degree one and subdivision of edges (by vertices), and the presence of such vertices obstructs passage to the limit. In this terminology, the $\phi^{4}$-model corresponds to the case in which only vertices of degree four are permitted, while the Penner model corresponds to the case in which there are no vertex restrictions, except for the suppression of vertices of degree one and two.

Random matrix models are useful in the study of random surfaces [27] and physical processes acting on them. Such surfaces occur in statistical mechanics where the underlying geometry may change. The critical behaviour of statistical mechanical models of unitary matrices as $N \rightarrow \infty$ and $\beta / N \rightarrow \lambda_{c}$ has been studied by Periwal and Shevitz [29], and critical phenomena have been studied by Distler and Vafa [7]. For details about these topics the reader is referred to Itzykson and Drouffe [19], Mehta [26] and Ginsparg [10].

1.3. The Main Integral $R(\mathbf{x}, N, z)$. The objects of study of this paper are the series $R_{4}(x, y, z)$ and $R_{\overline{1}}(x, y, z)$, and their relationship to graphs in surfaces. These completely determine the partition function $Z_{N}(U)$ for Hermitian-complex random matrix models, and also their relationship to graphs in surfaces. The series have been studied extensively, and are defined in terms of the main integral by

$$
\left.\begin{array}{rl}
R_{4}(x, y, z) & =R(\mathbf{x}, y, z) \\
R_{\overline{1}}(x, y, z) & \text { at } \mathbf{x}=(0,0,0, x, 0, \ldots) \\
\mathbf{x}, y, z) & \text { at } \mathbf{x}=(0,0, x, x, x, \ldots)
\end{array}\right\}
$$

where the main integral is

$$
R(\mathbf{x}, N, z)=\frac{1}{\sqrt{2 \pi}^{N}} \prod_{j=1}^{N} \frac{1}{j !} \cdot \int_{\mathbb{R}^{N}} V^{2}(\lambda) e^{\sum_{k \geqq 1} \frac{1}{k} \sqrt{z}^{k} x_{k} p_{k}} e^{-\frac{1}{2} p_{2}} d \lambda \in \mathbb{Q}[\mathbf{x}, N][[z]]
$$

in which $\lambda=\left(\lambda_{1}, \ldots, \lambda_{N}\right), p_{k}=\lambda_{1}^{k}+\cdots+\lambda_{N}^{k}, k$ is a positive integer, $y, z, x_{1}, x_{2}, \ldots$ are indeterminates, $\mathbf{x}=\left(x_{1}, x_{2}, \ldots\right)$, and $V(\lambda)$ is the Vandermonde determinant. The ring of formal power series in the indeterminate $z$ with coefficients that are polynomials, with rational coefficients, in the indeterminates $\mathbf{x}$ and $N$ is denoted by $\mathbb{Q}[\mathbf{x}, N][[z]] . R(\mathbf{x}, N, z)$ is a power series in $z$ with coefficients which are polynomial in $N$ and in $x_{1}, x_{2}, \ldots$, so $R(\mathbf{x}, y, z)$ is defined by replacing formally $N$ by $y$. Let $R_{\mathscr{N}}(x, y, z)=R(\mathbf{x}, y, z)$ at $\mathbf{x}=(x, x, \ldots)$. It is well known that the integral (1) can be transformed into one of the form (3). The usual observation $[26,32]$ is that the integrand is invariant under the adjoint action of the unitary group so, by unitary diagonalisation, the integral is replaced by one over the spectrum, which is real, and an integration over the unitary group, which is straightforward either by constructing Haar measure or by indirect computation. $Z_{N}(U), \mathscr{Z}_{N}(U, \beta)$, and $R(\mathbf{x}, N, z)$ are simply rewritings of essentially the same function. From this point onwards it will be convenient to refer exclusively to $R(\mathbf{x}, N, z)$.

Of parallel importance to matrix models are embeddings of graphs on surfaces. We therefore define these now, although they are not used until Sect. 3. 
1.4. Combinatorial Terminology. For consistency, throughout we have adopted combinatorial terminology for graphs and graph embeddings.

1.4.1. Graphs. An edge is an unordered pair of vertices of a graph as an incidence relation and also the line between the vertices in an embedding of the graph in the surface (the distinction is always clear from the context); a loop is an edge with end vertices identified; a multiple-edge is a collection of edges with initial vertices identified and terminal vertices identified; a surface is a two dimensional manifold. The number of edges incident with a vertex is called the degree of a vertex.

1.4.2. Embeddings. Let $G$ be a connected (unlabelled) graph, with $n$ edges, in which loops and multiple edges are allowed. Each edge is assigned a direction and a unique integer label between 1 and $n$. The set of origins $e^{+}$and termini $e^{-}$of edges $e$ in the edge-set $\mathrm{E}(G)=\{1, \ldots, n\}$ of $G$ is called the directed edge set of $G$. Let $\Sigma$ be a closed oriented surface without boundary. An embedding of $G$ in $\Sigma$ is a continuous injective function $\varepsilon: \mathscr{G} \rightarrow \Sigma$. Two embeddings $\varepsilon, \varepsilon^{\prime}$ are equivalent if there is an orientation preserving homeomorphism $\psi: \Sigma \rightarrow \Sigma$ such that $\psi \varepsilon=\varepsilon^{\prime}, \psi \varepsilon(e)=\varepsilon^{\prime}(e)$, and $\psi \varepsilon\left(e^{+}\right)=\varepsilon^{\prime}\left(e^{+}\right)$, for all $e \in\{1, \ldots, n\}$; that is, $\psi$ respects labelling and directing of edges. A map with associated graph $G$ is an embedding of $G$ in $\Sigma$. A map is a 2-cell embedding of a graph on a surface, in the sense that deletion of the graph separates the surface into regions homeomorphic to discs, called faces. The number of edges bounding a face is called the degree of a face. The map is rooted by distinguishing a mutually incident vertex, edge and face or, equivalently, by selecting an edge and assigning a direction to it. Rooting is a combinatorial artifice for accounting for the automorphisms of the embedding. Indeed, the number of inequivalent rootings divides twice the number of edges.

1.4.3. The Genus Series. Let $M(\mathbf{x}, \mathbf{y}, z)=\sum_{\mathbf{i}, \mathbf{j}, k} m_{\mathbf{i}, \mathbf{j}, k} x_{1}^{i_{1}} x_{2}^{i_{2}} \cdots y_{1}^{j_{1}} y_{2}^{j_{2}} \cdots z^{k}$, where $m_{\mathbf{i}, \mathbf{j}, k}$ is the number of rooted maps with $i_{1}$ vertices of degree $1, i_{2}$ vertices of degree $2, \ldots, j_{1}$ faces of degree $1, j_{2}$ faces of degree $2, \ldots$, and $k$ edges, and $\mathbf{i}=\left(i_{1}, \ldots\right)$, $\mathbf{j}=\left(j_{1}, \ldots\right)$. We say that $x_{j}$ marks vertices of degree $j$. From this information, the genus of the map, and therefore the genus of the surface is derivable by the EulerPoincare formula. Therefore, $M(\mathbf{x}, \mathbf{y}, z)$ is called the genus series for rooted maps (in orientable surfaces). Notice that monovalent and bivalent vertices are allowed in this formulation. Let $M_{\mathscr{N}}(x, y, z)=M(\mathbf{x}, \mathbf{y}, z)$, where $\mathbf{x}=(x, x, \ldots)$, and $\mathbf{y}=(y, y, \ldots)$, where $\mathscr{N}=\{1,2, \ldots\}$. This lists the set of permissible vertex degrees.

It may appear more natural to regard $\Gamma$ as the genus series, where

$$
\Gamma\left(u^{2}, \mathbf{x}, \mathbf{y}, z\right)=u^{2} M\left(u^{-1} \mathbf{x}, u^{-1} \mathbf{y}, u z\right),
$$

by the Euler-Poincare formula, and where $u$ is an indeterminate marking genus. In practice, this is less convenient. However, it raises the point that, although it is clear combinatorially that $\Gamma\left(u^{2}, \mathbf{x}, \mathbf{y}, z\right) \in \mathbb{Q}[u, \mathbf{x}, \mathbf{y}][[z]]$, this is evident neither from the integral representation (3), nor from the character representation (10) of the genus series.

1.5. The Approach. Apart from the assertion of the existence of $\int_{\mathbb{R}} e^{-\frac{1}{2} x^{2}} d x$, the results are established by an algebraic argument in an appropriate ring of formal power series that is clear from the context and will not be stated explicitly, to avoid cluttering the discussion with unnecessary detail. On occasion it will be necessary to 
establish that a coefficient ring is a polynomial ring, invariably to permit extension of a result from one over the integers to one in an indeterminate. Typically, the polynomiality devolves from local finiteness of combinatorial structures. Extensive use is made of the ring of symmetric functions, properties of the characters of irreducible representations of the symmetric group, and combinatorial constructions. The algebraic arguments rest on the combinatorial nature of these topics. Nevertheless, the formal power series can be examined for a domain of convergence, and the corresponding function can then be treated by analysis.

The integral representations rest on the work of 't Hooft [18] and Bessis, Itzykson and Zuber [3], although they arise here in an entirely different way. The interconnexion of combinatorial, algebraic and analytic material rests on the work of Jackson [20,21], and Jackson and Visentin [22-24] on embeddings in orientable surfaces. Such connexions have also been explored in a different context by Di Francesco and Itzykson [6].

1.6. The Main Theorems. Below are the statements of the main results and the supporting notation. The remainder of the paper is concerned with their proof.

\subsubsection{Factorisation of the Partition Function for Matrix Models.}

Theorem A.

$$
\begin{aligned}
R_{4}(x, y, z) & =R_{\mathscr{N}}\left(\frac{1}{2} x, \frac{1}{2}(x+1), 4 z^{2} y\right) \cdot R_{\mathscr{N}}\left(\frac{1}{2} x, \frac{1}{2}(x-1), 4 z^{2} y\right), \\
R_{4}(1,2 N, z) & =R_{\mathscr{N}}\left(N+\frac{1}{2}, N, 4 z^{2}\right) \cdot R_{\mathscr{N}}\left(N-\frac{1}{2}, N, 4 z^{2}\right) .
\end{aligned}
$$

Equation (4) of Theorem A gives a strikingly simple relationship between $R_{4}(x, y, z)$ and $R_{\mathscr{N}}(x, y, z)$, and therefore between two matrix models. In view of the close relation with $Z_{N}(U)$, we call Eq. (5) of Theorem A a factorisation associated with the partition function.

1.6.2. Combinatorial Maps. Let $\alpha_{1} \geqq \alpha_{2} \geqq \cdots \geqq 0$ be integers which sum to $k$. Then $\alpha=\left(\alpha_{1}, \alpha_{2}, \ldots\right)$ is called a partition of $k$, and we write $\alpha \vdash k$. The number of positive parts of $\alpha$ is its length and is denoted by $l(\alpha)$. The number of parts of $\alpha$ that are equal to $j$ is denoted by $m_{j}(\alpha)$. When this frequency information is available, we write $\alpha=\left[1^{m_{1}(\alpha)}, 2^{m_{2}(\alpha)}, \ldots\right]$. If $\alpha \vdash k$, let $h^{\alpha}=k ! / \prod_{j \geqq 1} j^{m_{j}(\alpha)} m_{j}(\alpha) !=k ! g(\alpha)$. We denote $x_{\alpha_{1}} x_{\alpha_{2}} \cdots$ by $x_{\alpha}$, and $k$ by $|\alpha|$. Let $\chi_{\alpha}^{\theta}$ denote the character $\chi^{\theta}$ of the irreducible ordinary representation of the symmetric group, indexed by the partition $\theta$, evaluated at the conjugacy class for which the partition $\alpha$ is a natural index. The size of this class is $h^{\alpha}$. Let $f^{\theta}=\chi_{\left[1^{2 n}\right]}^{\theta}$, the degree of the ordinary irreducible representation indexed by $\theta \vdash 2 n$. Let $H_{\theta}(y)$ be the polynomial in $y$ of degree $|\theta|$ defined by

$$
H_{\theta}(y)=\prod_{j=1}^{l(\theta)}(y-j+1)^{\left(\theta_{j}\right)},
$$

where $(y)^{(k)}=y(y+1) \cdots(y+k-1)$, the rising factorial function.

Theorem B gives the genus series for combinatorial maps. The series $R(\mathbf{x}, \mathbf{y}, z)$ defined in the theorem specialises to $R_{\mathscr{N}}(x, y, z)$, and this accounts for the choice of 
notation for this series. It may be thought of as giving an algebraic representation of the genus series, as opposed to the analytic one provided by (3) in conjunction with (8). Recall that $M(\mathbf{x}, \mathbf{y}, z)$ is the genus series for maps described earlier.

Theorem B. Let

Then

$$
R(\mathbf{x}, \mathbf{y}, z)=\sum_{n \geqq 0} \frac{z^{n}}{n !(2 n) !} \sum_{v, \phi \vdash 2 n} h^{v} h^{\phi} x_{v} y_{\phi} \sum_{\theta \vdash 2 n} \frac{1}{f^{\theta}} \chi_{\phi}^{\theta} \chi_{\nu}^{\theta} \chi_{\left[2^{n}\right]}^{\theta} .
$$

$$
\begin{aligned}
M(\mathbf{x}, \mathbf{y}, z) & =2 z \frac{\partial}{\partial z} \log R\left(\mathbf{x}, \mathbf{y}, \frac{1}{2} z\right), \\
R(\mathbf{x}, y, z) & =\sum_{n \geqq 0} \frac{z^{n}}{n !(2 n) !} \sum_{v \vdash 2 n} h^{v} x_{v} \sum_{\theta \vdash 2 n} \chi_{v}^{\theta} \chi_{\left[2^{n}\right]}^{\theta} H_{\theta}(y), \\
R_{\mathcal{N}}(x, y, z) & =\sum_{n \geqq 0} \frac{z^{n}}{n !(2 n) !} \sum_{\theta \vdash 2 n} f^{\theta} \chi_{\left[2^{n}\right]}^{\theta} H_{\theta}(x) H_{\theta}(y) .
\end{aligned}
$$

Note that (3) agrees with (9) at the positive integers.

1.6.3. Maps and Vertex 4-Regular Maps. The next theorem gives a simple relationship between the genus series $M_{\mathscr{N}}$ for all maps and the genus series $M_{4}$ for maps with vertices of degree four only. Such maps are called vertex 4regular. Although it is a direct consequence of Theorems $\mathrm{A}$ and $\mathrm{B}$, it is stated separately.

Theorem C. $\frac{1}{2} M_{4}(x, y, z)=M_{\mathscr{N}}\left(\frac{1}{2} x, \frac{1}{2}(x+1), 2 z^{2} y\right)+M_{\mathscr{N}}\left(\frac{1}{2} x, \frac{1}{2}(x-1), 2 z^{2} y\right)$.

1.6.4. A Relationship between the $\phi^{4}$ - and Penner-Models. Theorem D gives an explicit relation between the genus series for two important classes of maps. Let $M_{\overline{1}}$ be the genus series for maps with no vertices of degree one or two.

Theorem D. Let $s=\sqrt{1-4 x y z^{2}}$. Then

$$
\begin{aligned}
& \frac{1+s}{s^{2}}\left\{M_{\overline{1} \overline{2}}\left(\frac{x}{2}, \frac{x+1}{2}, \frac{4 y z^{2}}{s(1+s)}\right)+M_{\overline{1} \overline{2}}\left(\frac{x}{2}, \frac{x-1}{2}, \frac{4 y z^{2}}{s(1+s)}\right)\right\} \\
& =M_{4}(x, y, z)-\frac{2 x y z^{2}}{s(1+s)}\left(\frac{1+x^{2}}{s}-\frac{2 x}{1+s}+2 x^{2}\right) .
\end{aligned}
$$

In view of the discussion of Sect. 1.2, the relation given in Theorem $\mathrm{D}$ implies a connexion between a pair of matrix models and, equivalently, a connexion between the $\phi^{4}$-model (corresponding to $M_{4}$ ) and the Penner model (corresponding to $M_{\overline{1} \overline{2}}$ ), at least at the combinatorial level of graphs embedded on surfaces, before the double scaling limit has been taken. In this limit, it is the terms that are universal that are of interest, since these are believed to convey physical information.

It is a consequence of Theorem B that

$$
M_{\overline{1} \overline{2}}(\mathbf{x}, y, z)=2 z \frac{\partial}{\partial z} \log R_{\overline{1} \overline{2}}\left(\mathbf{x}, y, \frac{1}{2} z\right), \quad M_{4}(\mathbf{x}, y, z)=2 z \frac{\partial}{\partial z} \log R_{4}\left(\mathbf{x}, y, \frac{1}{2} z\right) .
$$


Thus, Theorem $\mathrm{D}$ can also be interpreted strictly as a result about the two integrals $R_{\overline{1} \overline{2}}$ and $R_{4}$, rather than a result about genus series.

1.6.5. A Reformulation for the $\phi^{4}$-Model. Theorem E concerns vertex-regular maps. A map is said to be vertex $k$-regular if every vertex has degree $k$, and regular if it is vertex $k$-regular for some integer $k$, regardless of the value of $k$.

The set of nonnegative integers is denoted by $\mathbb{N}$, and $X=\left(X_{1}, \ldots, X_{k}\right)$ and $u=\left(u_{1}, \ldots, u_{k}\right)$ are vectors of indeterminates. For convenience, we set $X_{0}=1$. For a vector $\sigma=\left(\sigma_{1}, \ldots, \sigma_{k}\right)$, of positive integers, $m_{j}(\sigma)$ is the number of occurrences of $j$ in $\sigma$. For $\alpha \in \mathbb{N}^{k}$, let $u^{\alpha}=u_{1}^{\alpha_{1}} \cdots u_{k}^{\alpha_{k}}$, and $\alpha !=\alpha_{1} ! \cdots \alpha_{k} !$, and $\mathbf{u}=\left(u_{1}, \ldots, u_{k}\right)$. Let

$$
\Psi_{k}(X, \mathbf{u})=\frac{1}{1-X_{k}} \exp \left\{\frac{1+X_{k}}{1-X_{k}} \frac{p_{2}(\mathbf{u})}{4}+\frac{1}{1-X_{k}} \sum_{1 \leqq r<s \leqq k}\left(\frac{X_{s}}{X_{r}}+X_{k} \frac{X_{r}}{X_{s}}\right) \frac{u_{r} u_{s}}{2}\right\} .
$$

Let $\left[X_{1}^{0} \cdots X_{k-1}^{0}\right] f(X, u)$ denote the constant term of $f$ in $X_{1}, \ldots, X_{k-1}$, namely, the subseries of $f$ of terms independent of $X_{1}, \ldots, X_{k-1}$.

Theorem E. Let $\alpha \in \mathbb{N}^{k}$ and let $P_{\alpha}(N)$, a polynomial in $N$, be such that

$$
\sum_{\alpha \in \mathbb{N}^{k}, N \geqq 1} P_{\alpha}(N) \frac{u^{\alpha}}{\alpha !} X_{k}^{N-1}=\left[X_{1}^{0} \cdots X_{k-1}^{0}\right] \Psi_{k}(X, \mathbf{u}) \prod_{i=1}^{k}\left(1-\frac{X_{i}}{X_{i-1}}\right)^{-1} .
$$

Let $n$ be a positive integer or a half-integer. Then the genus series for $2 n$-regular maps on $k$ vertices and $n k$ edges, with respect to faces (marked by $y$ ), is

$$
\frac{2^{n k} k}{(2 n)^{k-1}} F_{n, k}(y) \quad \text { where } F_{n, k}(y)=\sum_{\alpha \vdash k} \frac{(-1)^{l(\alpha)-1}}{l(\alpha)} \frac{1}{\alpha ! m(\alpha) !} \sum_{\sigma \in \subseteq_{k}} P_{2 n \sigma(\alpha)}(y) .
$$

In fact, as will be seen, $P_{\alpha}(N)$ and $F_{n, k}(N)$ are polynomials in $N$, so $P_{\alpha}(y)$ and $F_{n, k}(y)$ are defined by formally replacing $N$ by $y$.

Theorem E expresses the genus series for regular maps as the constant term in a multivariate series. These maps are of interest since their genus series contains the genus series $4 k F_{2, k}(y)$ for 4-regular maps and these, as has been observed, are associated with the $\phi^{4}$-model.

Although we have not succeeded in deriving an explicit expression for the genus series itself, the form obtained is a remarkably elementary one which fully captures information about genus. We observe that the expression presented here does not require further integration, and so in some sense represents a solution of the $\phi^{4}$-model.

1.6.6. A Factorisation for Hypermaps. A p-face-colourable map is a map whose faces may be assigned one of $p$ colours such that no edge separates faces of the same colour. The colour assigned to the root face is called the root colour. The root face is the face on the (conventionally) left-hand side of the edge selected to determine the root, given that one is looking in the direction of the orientation. For a 2-face-coloured map, the other colour is the non-root colour, and interchange of the root-face-colour corresponds to the reversal of the direction of the root edge. A 2-face-coloured map is also called a hypermap. 
Let $M_{\mathscr{A}}(x, y, z)$ be the genus series for maps with no vertex of degree not in the subset $\mathscr{A}$ of the positive integers. Let $C_{\mathscr{A}_{1}, \mathscr{A}_{2}}\left(x, y_{1}, y_{2}, z\right)$ be the genus series for rooted 2-face-coloured maps with no root-coloured faces of degree not in $\mathscr{A}_{1}$, no non-root-coloured faces of degree not in $\mathscr{A}_{2}$, and in which $x, y_{1}, y_{2}, z$ mark, respectively, the numbers of vertices, faces of the root colour, faces of the nonroot colour, and edges.

\section{Theorem F.}

$\frac{1}{2} M_{\{2 r\}}(x, y, z)=C_{\{r\}, \mathcal{N}}\left(\frac{1}{2}(x+1), \frac{1}{2} y, \frac{1}{2} x, 2 z\right)+C_{\{r\}, \mathcal{N}}\left(\frac{1}{2}(x-1), \frac{1}{2} y, \frac{1}{2} x, 2 z\right)$.

Theorem $\mathrm{F}$ therefore states a relationship between the 1-matrix model and other matrix models.

\section{Factorisation of the Integral}

The purpose of this section is to prove the factorisation theorem entirely independently of the idea of a surface. The proof is essentially an algebraic one. The reader is referred to Macdonald [25] for the details and proofs of classical results about symmetric functions, and to Serre [30]. If $f$ is a formal power series in $x$, let $\left[x^{n}\right] f$ denote the coefficient of $x^{n}$ in $f$, whenever $n$ is a non-negative integer.

For $\theta \vdash n$, let $\chi^{\theta}$ be the character of the ordinary irreducible representation of the symmetric group $\Xi_{n}$, indexed by $\theta$, and let $\chi_{\alpha}^{\theta}$ denote the value of this function at an arbitrary element in the conjugacy class indexed (naturally) by $\alpha \vdash n$. The size of the conjugacy class indexed by $\alpha$ is denoted by $h^{\alpha}$ and is equal to $n ! g(\alpha)$, where

$$
g(\alpha)=\frac{1}{\prod_{j \geqq 1} j^{m_{j}(\alpha)} m_{j}(\alpha) !},
$$

where $\alpha=\left(\alpha_{1}, \alpha_{2}, \ldots\right)$.

2.1. The Character Formulation of the Integral. The first step of the proof of Theorem $\mathrm{A}$ is to transform the integral representation of $R$ into a sum over characters of irreducible representations of the symmetric group. Let $p_{\alpha}$ denote $p_{\alpha_{1}} p_{\alpha_{2}} \ldots$, where the power sum symmetric function $p_{k}$, for a nonnegative integer $k$, has been defined in Sect. 1.3. By expanding $\exp \left(\sum_{k \geqq 1} \frac{1}{k} \sqrt{z}^{k} x_{k} p_{k}\right)$ in (3), $R$ can be rewritten in the form

$$
\begin{aligned}
R(\mathbf{x}, N, z) & =\sum_{k \geqq 0} \frac{2^{k} z^{k}}{(2 k) !} \sum_{\alpha \vdash 2 k} h^{\alpha} A_{\alpha}(N) x_{\alpha}, \\
A_{\alpha}(N) & =\frac{1}{\sqrt{2 \pi}^{N}} \frac{1}{\prod_{j=1}^{N} j !} \int_{\mathbb{R}^{N}} V^{2}(\lambda) p_{\alpha} e^{-\frac{1}{2} p_{2}} d \lambda .
\end{aligned}
$$

The Schur function $s_{\theta}$ is given in terms of the power sums by

$$
s_{\theta}=\sum_{\gamma \vdash n} g(\gamma) \chi_{\gamma}^{\theta} p_{\gamma},
$$


a result due to Frobenius. We therefore begin by considering the integral

$$
\mathscr{I}_{\theta}(N)=\frac{1}{\sqrt{2 \pi}^{N}} \frac{1}{N !} \int_{\mathbb{R}^{N}} V^{2}(\lambda) s_{\theta} e^{-\frac{1}{2} p_{2}} d \lambda
$$

since, through the orthogonality $\sum_{\theta \vdash n} \chi_{\alpha}^{\theta} \chi_{\beta}^{\theta}=g^{-1}(\alpha) \delta_{\alpha, \beta}$ of the characters, and (16) and (17), $A_{\alpha}$ has a simple expression in terms of $\mathscr{I}_{\theta}$, namely

$$
A_{\alpha}(N)=\frac{1}{2^{n} n !} \sum_{\theta \vdash 2 n} \mathscr{I}_{\theta}(N) \chi_{\alpha}^{\theta}
$$

The integral for $\mathscr{I}_{\theta}$ can be determined as follows. Let $a_{\theta}=\operatorname{det}\left[x_{i}^{\theta_{j}}\right]_{N \times N}$, so the Vandermonde determinant in this notation is $V(\lambda)=a_{\delta}$, where $\delta=(N-1$, $N-2, \ldots, 1,0)$. But $s_{\theta}=a_{\theta+\delta} / a_{\delta}$, a quotient of alternants, so $V^{2}(\lambda) s_{\theta}=a_{\theta+\delta} a_{\delta}$, where $N \geqq l(\theta)$. The determinants can now be expanded to give

$$
V^{2}(\lambda) s_{\theta}=\sum_{\sigma, \rho \in \subseteq_{N}} \operatorname{sgn}(\sigma \rho) \prod_{i=1}^{N} \lambda_{i}^{2 N+\theta_{\sigma(i)}-\sigma(i)-\rho(i)} .
$$

The product can be reordered by setting $j=\sigma(i)$, so from (18),

$$
\begin{aligned}
\mathscr{I}_{\theta}(N) & =\frac{1}{\sqrt{2 \pi}^{N}} \frac{1}{N !} \int_{\mathbb{R}^{N}} \sum_{\sigma, \rho \in \Im_{N}} \operatorname{sgn}(\sigma \rho)\left(\prod_{j=1}^{N} \lambda_{\sigma^{-1}(j)}^{2 N+\theta_{j}-j-\rho \sigma^{-1}(j)}\right) e^{-\frac{1}{2} p_{2}} d \lambda \\
& =\frac{1}{N !} \sum_{\sigma, \rho \in \Im_{N}} \operatorname{sgn}(\sigma \rho) \prod_{j=1}^{N}\left(\frac{1}{\sqrt{2 \pi}} \int_{\mathbb{R}} t^{2 N+\theta_{j}-j-\rho \sigma^{-1}(j)} e^{-\frac{1}{2} t^{2}} d t\right)
\end{aligned}
$$

on rearranging the order of the integration. Let $\phi=\rho \sigma^{-1}$. Since sgn is a character of $\mathfrak{\Im}_{N}$, then $\operatorname{sgn}(\phi)=\operatorname{sgn}(\sigma \rho)$, so

$$
\mathscr{I}_{\theta}(N)=\frac{1}{N !} \sum_{\phi \in \mathfrak{S}_{N}} \operatorname{sgn}(\phi) \prod_{j=1}^{N}\left(\frac{1}{\sqrt{2 \pi}} \int_{\mathbb{R}} t^{2 N+\theta_{j}-j-\phi(j)} e^{-\frac{1}{2} t^{2}} d t\right) \sum_{\substack{\sigma, \rho \in \mathfrak{S}_{N} \\ \rho \sigma^{-1}=\phi}} 1 .
$$

But, given $\phi$, the equation $\rho \sigma^{-1}=\phi$ has a unique solution $\rho$ for each $\sigma$, so the combinatorial sum in (20) over $\sigma, \rho$ is easily determined to be $N$ !, independent of $\phi$. Let

$$
\langle j\rangle=\frac{1}{\sqrt{2 \pi}} \int_{\mathbb{R}} t^{j} e^{-t^{2} / 2} d t,
$$

where $j$ is a nonnegative integer. Then $\langle 2 k\rangle=h^{\left[2^{k}\right]}$ if $k$ is a nonnegative integer, and $\langle k\rangle=0$ if $k$ is an odd or negative integer. Thus, from (20),

$$
\mathscr{I}_{\theta}(N)=\sum_{\phi \in \subseteq_{N}} \operatorname{sgn}(\phi) \prod_{j=1}^{N}\left\langle 2 N+\theta_{j}-j-\phi(j)\right\rangle=\operatorname{det}\left(\left[\left\langle 2 N+\theta_{i}-i-j\right\rangle\right]_{N \times N}\right) .
$$

The evaluation of $\mathscr{I}_{\theta}(N)$ is completed by expanding and identifying this determinant in terms of characters of $\mathfrak{\Xi}_{N}$. Let $q$ be a nonnegative integer and $n$ a positive integer. Under the identification $n=N-j$ and $q=\theta_{i}+j$ we have $\langle q+2 n-2 k\rangle=\left\langle 2 N+\theta_{i}-i-(j+2 k)\right\rangle$, the $(i, j+2 k)^{\text {th }}$ element of the matrix whose determinant is to be determined. We seek to simplify the determinant by 
using a series of column operations. From (21), a recurrence equation for $\langle q\rangle$ is $\langle q+2\rangle=(q+1)\langle q\rangle$, so $\langle q+2 n\rangle /\langle q\rangle$ is a polynomial of degree $n$ in $q$, and $(q+1)(q+2) \cdots(q+n)$ is therefore a unique linear combination of $\langle q+2 j\rangle /\langle q\rangle$ for $0 \leqq j \leqq\lfloor n / 2\rfloor$. The linear combination is obtained by observing that $\left[t^{q}\right](2+t)^{q+n}=\left[t^{2 q+n}\right](1+t)^{2 q+2 n}\left(1-(1+t)^{-2}\right)^{q+n}$ so

$$
2^{n}\left(\begin{array}{c}
q+n \\
n
\end{array}\right)=\sum_{k \geqq 0}(-1)^{k}\left(\begin{array}{c}
q+n \\
k
\end{array}\right)\left(\begin{array}{c}
2(q+n-k) \\
2 q+n
\end{array}\right) .
$$

But from the recurrence equation for $\langle q\rangle$,

$$
\langle 2 q+2 n-2 k\rangle=\frac{1}{2^{n-k}} \frac{q !}{(2 q) !} \frac{(2 q+2 n-2 k) !}{(q+n-k) !}\langle 2 q\rangle,
$$

so (23) becomes $\langle 2 q\rangle(2 q+1)(2 q+2) \cdots(2 q+n)=\sum_{k=0}^{\lfloor n / 2\rfloor} c_{n, k}\langle 2 q+2 n-2 k\rangle$, where $c_{n, k}=(-1)^{k}\langle 2 k\rangle\left(\begin{array}{c}n \\ 2 k\end{array}\right)$. But $\langle q\rangle$ and $\langle q+2 n-2 k\rangle$ are 0 when $q$ is odd, so

$$
\langle q\rangle(q+1)(q+2) \cdots(q+n)=\sum_{k=0}^{\lfloor n / 2\rfloor} c_{n, k}\langle q+2 n-2 k\rangle .
$$

Now consider the matrix $\left[\left\langle 2 N+\theta_{i}-i-j\right\rangle\right]_{N \times N}$. Its determinant is unchanged by the addition of a multiple of column $j+2 k$ to column $j$, for $k=1, \ldots$, $\lfloor(N-j) / 2\rfloor$. The multiple that is selected is $c_{N-j, k}$. Let $\theta_{i, j}=\theta_{i}-i+j$. Then, from $(22)$,

$$
\mathscr{I}_{\theta}(N)=\operatorname{det}\left(\left[\sum_{k=0}^{\lfloor(N-j) / 2\rfloor} c_{N-j, k}\left\langle\theta_{i, j}+2(N-j)-2 k\right\rangle\right]_{N \times N}\right),
$$

having noted that $c_{N-j, 0}=1$. Then from (24),

$$
\mathscr{I}_{\theta}(N)=\operatorname{det}\left(\left[\left\langle\theta_{i, j}\right\rangle\left(\theta_{i, j}+1\right)^{(N-j)}\right]_{N \times N}\right)=H_{\theta}(N) \cdot \operatorname{det}\left(\left[\frac{\left\langle\theta_{i, j}\right\rangle}{\theta_{i, j} !}\right]_{N \times N}\right),
$$

where $H_{\theta}(y)$ is the polynomial in $y$ of degree $|\theta|$ defined in (6).

The remaining determinant can be evaluated in terms of certain characters of the symmetric group. Let $w=\left(w_{1}, w_{2}, \ldots\right)$, where $w_{1}, w_{2}, \ldots$ are indeterminates. The complete symmetric functions $h_{k}(w)$ are defined by $\sum_{k \geqq 0} h_{k}(w) t^{k}=$ $\prod_{k \geqq 1}\left(1-w_{k} t\right)^{-1}$, and are extended multiplicatively by $h_{\theta}=h_{\theta_{1}} h_{\theta_{2}} \cdots$. The $h_{\theta}(w)$ are algebraically independent. Similarly, the $p_{\theta}$ are algebraically independent. But $h_{r}(w)=\sum_{\alpha \vdash r} g(\alpha) p_{\alpha}(w)$. Moreover, $h^{\left[2^{r}\right]}=(2 r) ! g\left(\left[2^{r}\right]\right)$. If $f$ is a symmetric function, let $\left[p_{v}\right] f$ denote the coefficient of $p_{v}$ in $f$. Since the power sums afford a basis of the ring of symmetric functions, this operation is well defined. Thus

$$
\begin{aligned}
\operatorname{det}\left(\left[\frac{\left\langle\theta_{i, j}\right\rangle}{\theta_{i, j} !}\right]_{N \times N}\right) & =\operatorname{det}\left(\left[\left[p_{2}^{\left(\theta_{i}-i+j\right) / 2}(w)\right] h_{\theta_{i}-i+j}(w)\right]\right) \\
& =\left[p_{2}^{n}(w)\right]\left(\operatorname{det}\left[h_{\theta_{l, j}}(w)\right]_{l(\theta) \times l(\theta)}\right)
\end{aligned}
$$


since the $p_{i}(w)$ are algebraically independent, and $\theta \vdash 2 n$. By the Jacobi-Trudi theorem

$$
s_{\theta}(w)=\operatorname{det}\left(\left[h_{\theta_{J}-i+j}(w)\right]_{l(\theta) \times l(\theta)}\right),
$$

so

$$
\operatorname{det}\left(\left[\frac{\left\langle\theta_{i, j}\right\rangle}{\theta_{i, j} !}\right]_{N \times N}\right)=\frac{1}{2^{n} n !} \chi_{\left[2^{n}\right]}^{\theta}
$$

It follows from (25) that $H_{\theta}(N) \chi_{\left[2^{n}\right]}^{\theta}=2^{n} n ! \mathscr{I}_{\theta}(N) / \prod_{j=1}^{N-1} j$ !, so, from (19), $A_{\alpha}(N)$ is exhibited as an explicit polynomial in $N$, which may then be replaced throughout by $y$ to obtain

$$
A_{\alpha}(y)=\frac{h^{\left[2^{k}\right]}}{(2 k) !} \sum_{\theta \vdash 2 k} H_{\theta}(y) \chi_{\alpha}^{\theta} \chi_{\left[2^{k}\right]}^{\theta} .
$$

Combining this with (3) and (15) gives the explicit expression (9) of Theorem B for $R$. In this representation of the series $R$, the integration has been fully carried out and has been replaced by a sum of characters. Moreover, in this formulation the original definition of $R(\mathbf{x}, N, z)$ extends to a natural definition of $R(\mathbf{x}, y, z)$. Properties of characters of irreducible representations can be used to obtain further properties of $R$, and this is done next.

2.2. Factorisation of Characters. Two preliminary results are needed. The first result simplifies (9) for the particular instance $R_{\mathscr{N}}(x, y, z)$ that concerns us here. It states that

$$
\sum_{\alpha \vdash N} h^{\alpha} \chi_{\alpha}^{\theta} x^{l(\alpha)}=f^{\theta} H_{\theta}(x)
$$

where $f^{\theta}=\chi_{\left[1^{2 n}\right]}^{\theta}$ is the degree result of the ordinary irreducible representation indexed by $\theta \vdash N$. In view of (2), an immediate consequence of (28) is a simplification of the expression for $R(\mathbf{x}, y, z)$ given in (9) to obtain $R_{\mathscr{N}}(x, y, z)$ in the form (10) of Theorem B. It is this form of $R_{\mathcal{N}}(x, y, z)$ that will be used to obtain its factorisation.

The second result is a factorisation of $\chi_{\left[(a b)^{n}\right]}^{\theta}$. First we derive a bijection between two combinatorial sets. Let $k, n$ be positive integers. For $\alpha=\left(\alpha_{1}, \alpha_{2}, \ldots\right) \vdash k n$ we define $\alpha^{\wedge}=\left(\alpha^{(1)}, \ldots, \alpha^{(k)}\right)$ by

$$
\left.\begin{array}{l}
\text { 1) }\left\{\eta_{j, 1}, \ldots, \eta_{j, m_{j}}\right\}=\left\{i: \alpha_{i}-i \equiv-j \bmod k\right\}_{<} \text {for } j=1, \ldots, k, \\
\text { 2) } \alpha^{(j)}=\left(\alpha_{1}^{(j)}, \ldots, \alpha_{m_{j}}^{(j)}\right) \text { for } j=1, \ldots, k, \\
\text { 3) } \alpha_{i}^{(j)}=\frac{1}{k}\left(\alpha_{\eta_{j, l}}-\eta_{j, i}+j\right)+i-1 \text { for } i=1, \ldots, m_{j} .
\end{array}\right\}
$$

If $m_{j}=\lceil(l(\alpha)-j+1) / k\rceil$ for all $j=1, \ldots, k$ then $\alpha$ is said to be $k$-balanced. Let $\mathscr{B}_{k, n}$ be the set of all $k$-balanced partitions of $k n$ and let $\Pi_{k, n}$ be the set of all sequences $\left(\theta^{(1)}, \ldots, \theta^{(k)}\right)$ of partitions whose weights sum to $n$. Clearly, for $\alpha$ with $l(\alpha)=q k+r$, where $q, r$ are integers and $0 \leqq r<k, \alpha$ is $k$-balanced if $m_{1}=\cdots=$ $m_{r}=q+1$ and $m_{r+1}=\cdots=m_{k}=q$. It can be shown by a case analysis that the mapping

$$
\Delta_{k}: \mathscr{B}_{k, n} \rightarrow \Pi_{k, n}: \theta \mapsto \theta^{\wedge}
$$


is a bijection. We call $\Delta_{k}(\theta)$ the (ordered) $k$-factorisation of $\theta \in \mathscr{B}_{k, n}$. Let $\theta$ be $k$-balanced and $\left(\theta^{(1)}, \ldots, \theta^{(k)}\right)$ be its $k$-factorisation. Then

$$
\left.\begin{array}{l}
k \text { divides }|\theta|, \\
H_{1}^{\theta}(x)=H_{k}^{\theta^{(1)}}(x) H_{k}^{\theta^{(2)}}(x-1) \cdots H_{k}^{\theta^{(k)}}(x-k+1), \\
H_{k}^{\theta}(x)=\prod_{1 \leqq i \leqq l(\theta)}(x-k i+k)^{\left(k \theta_{i}\right)} .
\end{array}\right\}
$$

The associated permutations of $\alpha^{\wedge}$ are $\pi_{\alpha}, \pi_{\alpha}^{\prime} \in \mathfrak{S}_{l(\alpha)}$ defined by

$$
\begin{aligned}
\pi_{\alpha} & =\left(\begin{array}{cccccc}
1 & \cdots & m_{1} & m_{1}+1 & \cdots & l(\alpha) \\
\eta_{1,1} & \cdots & \eta_{1, m_{1}} & \eta_{2,1} & \cdots & \eta_{k, m_{k}}
\end{array}\right), \\
\pi_{\alpha}^{\prime} & =\left(\begin{array}{cccccccc}
1 & 2 & 3 & \cdots & \cdots & l(\alpha) \\
1 & k+1 & 2 k+1 & \cdots & 2 & k+2 & \cdots &
\end{array}\right),
\end{aligned}
$$

where $\eta_{s, t}$ and $m_{t}$ are given in (1) of (29).

With these preliminary results we may now give a factorisation of $\chi_{\left[(a b)^{n}\right]}^{\theta}$. Let $\theta \vdash$ $a b n$. The mapping $\Xi_{k}: \Lambda \rightarrow \mathbb{Q}[[t]]: p_{\alpha} \mapsto t^{k r} \delta_{\alpha,\left[k^{r}\right]}$ extended linearly to $\Lambda$, is a ring homomorphism, since the $p_{\alpha}$ 's are algebraically independent. Thus, from (17) and (26) we have $\chi_{\left[(a b)^{n}\right]}^{\theta}=g^{-1}\left(\left[(a b)^{n}\right]\right)\left[p_{a b}^{n}\right] s_{\theta}=(a b)^{n} n !\left[t^{n a b}\right]\left\|\Xi_{a b} h_{\theta_{i}-i+j}\right\|_{l(\theta) \times l(\theta)}$. But $\Xi_{a b} h_{r}=t^{r}(a b)^{\frac{r}{a b}}\left(\frac{r}{a b}\right) !^{-1} \tau_{a b}(r)$, where $\tau_{j}(r)=1$ if $r \equiv 0 \bmod j$ and is 0 otherwise. Since $\theta \vdash a b n$,

$$
\chi_{\left[(a b)^{n}\right]}^{\theta}=n !|\mathbf{M}|, \quad \text { where } \quad \mathbf{M}=\left\|\tau_{a b}\left(\theta_{i}-i+j\right) /\left(\frac{\theta_{i}-i+j}{a b}\right) !\right\|_{l(\theta) \times l(\theta)} .
$$

Whether or not $\theta$ is $a$-balanced, we can still define $\theta^{\wedge}=\left(\theta^{(1)}, \ldots, \theta^{(a)}\right)$ by setting $k=a$ in (29). Thus $m_{s}$ and $\eta_{i, s}$ are determined. The permutation $\rho=\pi_{\theta}^{\prime}$ acting on the rows of this matrix and $\sigma=\pi_{\theta}$ acting on its columns rearrange the matrix as a direct sum while preserving the determinant, of course, up to sign. Thus $\chi_{\left[(a b)^{n}\right]}^{\theta}=$ $\operatorname{sgn}(\sigma \rho) n !\left|\mathbf{M}_{1} \oplus \cdots \oplus \mathbf{M}_{a}\right|$, where

$$
\left[\mathbf{M}_{1} \oplus \cdots \oplus \mathbf{M}_{a}\right]_{i j}=\tau_{a b}\left(\theta_{\rho(i)}-\rho(i)+\sigma(j)\right) /\left(\frac{\theta_{\rho(i)}-\rho(i)+\sigma(j)}{a b}\right) !
$$

for $1 \leqq i, j \leqq l(\theta)$, and $\mathbf{M}_{s}$ is $m_{s} \times\lceil(l(\theta)-s+1) / a\rceil$. To see this, it is sufficient to note that $\tau_{a b}\left(\theta_{\rho(i)}-\rho(i)+\sigma(j)\right)=0$ for any $(i, j)$ not corresponding to an element position in one of the blocks $\mathbf{M}_{1}, \ldots, \mathbf{M}_{a}$ of the direct sum, since $\theta_{\rho(i)}-\rho(i)+$ $\sigma(j) \neq 0$ mod $a$ for such values of $(i, j)$.

If $\theta$ is $a$-balanced, then $\mathbf{M}_{s}$ is square so $\chi_{\left[(a b)^{n}\right]}^{\theta}=\operatorname{sgn}(\sigma \rho) n ! \prod_{s=1}^{a}\left|\mathbf{M}_{s}\right|$, where

$$
\left[\mathbf{M}_{s}\right]_{i k}=\tau_{b}\left(\theta_{i}^{(s)}-i+k\right) /\left(\frac{\theta_{i}^{(s)}-i+k}{b}\right) !
$$

Since $\mathbf{M}_{s}$ has the same form as $\mathbf{M}$, we can evaluate $\left|\mathbf{M}_{s}\right|$ by trying to find a $b$ factorisation of $\theta^{(s)}$ and then using its associated permutations to rearrange $\mathbf{M}_{s}$. If $n_{s} \notin \mathcal{N}$, then from (31), $\theta^{(s)}$ is not $b$-balanced. Then using the same argument that was used to show that $|\mathbf{M}|=0$ when $\theta$ is not $a$-balanced, we have $\left|\mathbf{M}_{s}\right|=0$. 
If $n_{s} \in \mathscr{N}$, then $\left|\mathbf{M}_{s}\right|=n_{s} !^{-1} \chi_{\left[b^{n_{s}}\right]}^{\theta^{(s)}}$ from (32). We have therefore proved that, for $\theta \vdash a b n$,

$$
\chi_{\left[(a b)^{n}\right]}^{\theta}= \begin{cases}0 & \text { if } \theta \text { is not } a \text {-balanced }, \\ \operatorname{sgn}\left(\pi_{\theta} \pi_{\theta}^{\prime}\right) n ! \prod_{s=1}^{a} \frac{1}{n_{s} !} \chi_{\left[b^{n_{s}}\right]}^{\theta^{(s)}} & \text { otherwise }\end{cases}
$$

where $\Delta_{a}(\theta)=\left(\theta^{(1)}, \ldots, \theta^{(a)}\right)$ with associated permutations $\pi_{\theta}, \pi_{\theta}^{\prime}$, and $n_{s}=\left|\theta^{(s)}\right| / b$ for $s=1, \ldots, a$, with the convention that $\chi_{\left[b^{n_{s}}\right]}^{\theta^{(s)}}=0$ if $n_{s} \notin \mathscr{N}$.

2.3. The Factorisation of $R_{4}(x, y, z)$. We can now complete the proof of Theorem A. From (9)

$$
R_{4}(x, y, z)=\sum_{n \geqq 0} \frac{z^{2 n}}{(2 n) !} \frac{y^{n}}{4^{n} n !} \sum_{\theta \vdash 4 n} \chi_{\left[4^{n}\right]}^{0} \chi_{\left[2^{2 n}\right]}^{\theta} H_{\theta}(x) .
$$

Let $\left(\theta^{(1)}, \theta^{(2)}\right)$ be the 2-factorisation of $\theta$. From (33), the second summation may be restricted to 2-balanced $\theta$ for which $\left|\theta^{(1)}\right|$ and $\left|\theta^{(2)}\right|$ are even, for otherwise $\chi_{\left[4^{n}\right]}^{\theta}=0$. With $\left|\theta^{(1)}\right|=2 k$ and $\left|\theta^{(2)}\right|=2 n-2 k$, (33) gives

$$
\chi_{\left[4^{n}\right]}^{\theta}=\operatorname{sgn}\left(\pi_{\theta} \pi_{\theta}^{\prime}\right)\left(\begin{array}{l}
n \\
k
\end{array}\right) \chi_{\left[2^{k}\right]}^{\theta^{(1)}} \chi_{\left[2^{\left.2^{n-k}\right]}\right.}^{(2)}, \quad \chi_{\left[2^{2 n}\right]}^{\theta}=\operatorname{sgn}\left(\pi_{\theta} \pi_{\theta}^{\prime}\right)\left(\begin{array}{l}
2 n \\
2 k
\end{array}\right) f^{\theta^{(1)}} f^{\theta^{(2)}} .
$$

Moreover, from (31), $\prod_{1 \leqq i \leqq l(\theta)}(x-i+1)^{\left(\theta_{i}\right)}=H_{2}^{\theta^{(1)}}(x) H_{2}^{\theta^{(2)}}(x-1)$. It is known from (30) that $\Delta_{2}: \mathscr{B}_{2, n} \rightarrow \Pi_{2, n}$ is a bijection, so the above summation for $R_{4}(x, y, z)$ can now be transformed into

$$
\sum_{n \geqq 0} \frac{z^{2 n}}{(2 n) !} \frac{y^{n}}{4^{n} n !} \sum_{k=0}^{n}\left(\begin{array}{l}
n \\
k
\end{array}\right)\left(\begin{array}{l}
2 n \\
2 k
\end{array}\right) \sum_{\substack{\theta^{(1)}-2 k \\
\theta^{(2)} \vdash 2 n-2 k}} \chi_{\left[2^{k}\right]}^{\theta^{(1)}} \chi_{\left[2^{n-k}\right]}^{\theta^{(2)}} f^{\theta^{(1)}} f^{\theta^{(2)}} H_{2}^{\theta^{(1)}}(x) H_{2}^{\theta^{(2)}}(x-1) .
$$

Thus

$$
R_{4}(x, y, z)=G(x, y, z) G(x-1, y, z)
$$

where

$$
G(x, y, z)=\sum_{\substack{\theta \\|\theta| \equiv 0 \bmod 2}} \frac{z^{|\theta|} y^{\frac{1}{2}|\theta|}}{2^{|\theta|}\left(\frac{1}{2}|\theta|\right) !|\theta| !} \chi_{\left[2^{\frac{1}{2}|\theta|}\right]}^{\theta} f^{\theta} H_{2}^{\theta}(x) .
$$

To identify $G(x, y, z)$, it suffices to note that $H_{2}^{\theta}(x)$ can be rewritten in the particular form $H_{2}^{\theta}(x)=\prod_{1 \leqq i \leqq l(\theta)} 2^{2 \theta_{i}}\left(\frac{1}{2} x-i+1\right)^{\left(\theta_{i}\right)}\left(\frac{1}{2}(x+1)-i+1\right)^{\left(\theta_{i}\right)}$ so, from (6) and (10), $G(x, y, z)=R_{\mathscr{N}}\left(\frac{1}{2} x, \frac{1}{2}(x+1), 4 z^{2} y\right)$. Thus

$$
R_{4}(x, y, z)=R_{\mathscr{N}}\left(\frac{1}{2} x, \frac{1}{2}(x+1), 4 z^{2} y\right) \cdot R_{\mathscr{N}}\left(\frac{1}{2}(x-1), \frac{1}{2} x, 4 z^{2} y\right)
$$

from (34). But from (10) $R_{\mathscr{N}}\left(\frac{1}{2}(x-1), \frac{1}{2} x, 4 z^{2} y\right)=R_{\mathscr{N}}\left(\frac{1}{2} x, \frac{1}{2}(x-1), 4 z^{2} y\right)$ and (4) of Theorem A now follows.

But from (7) and (28),

$$
R_{4}(x, y, z)=\sum_{n \geqq 0} \frac{z^{2 n}}{(2 n) !} \frac{x^{n}}{4^{n} n !} \sum_{\theta \vdash 4 n} \chi_{\left[4^{n}\right]}^{\theta} \chi_{\left[2^{2 n}\right]}^{\theta} H_{\theta}(y),
$$


so $R_{4}(x, y, z)=R_{\mathscr{N}}\left(\frac{1}{2}(y+1), \frac{1}{2} y, 4 z^{2} x\right) \cdot R_{\mathscr{N}}\left(\frac{1}{2}(y-1), \frac{1}{2} y, 4 z^{2} x\right)$, on applying the above argument. But from (35), $R_{4}(x, y, z)=R_{4}\left(1, y, \sqrt{z^{2} x}\right)$. Thus replacing $z^{2} x$ by $z^{2}$ we have $R_{4}(1, y, z)=R_{\mathscr{N}}\left(\frac{1}{2}(y+1), \frac{1}{2} y, 4 z^{2}\right) \cdot R_{\mathscr{N}}\left(\frac{1}{2}(y-1), \frac{1}{2} y, 4 z^{2}\right)$, and (5) of Theorem A follows with $y=2 N$.

2.4. Permutations and $R_{\mathscr{N}}(x, y, z)$. We now rephrase $R(\mathbf{x}, y, z)$ defined in (9) by expressing the character sum as a consequence of operations in the group algebra of the symmetric group. This involves products of permutations, and we then seek a combinatorial configuration that can be encoded as a permutation, and for which the appropriate products of permutations have natural combinatorial interpretations. We begin with a generalisation of $R(\mathbf{x}, N, z)$ to $R(\mathbf{x}, \mathbf{y}, z)$, where $\mathbf{y}=\left(y_{1}, y_{2}, \ldots\right)$ are indeterminates, that is suggested indirectly by (9). In view of (9) and (28), Eq. (7) is a natural definition for $R(\mathbf{x}, \mathbf{y}, z)$. This is symmetric in $\mathbf{x}$ and $\mathbf{y}$, a fact that will be given a combinatorial explanation. Moreover, $R(\mathbf{x}, y, z)$ is recoverable from $R(\mathbf{x}, \mathbf{y}, z)$ by setting $y_{1}=y_{2}=\cdots=y$.

For $\alpha \vdash n$, let $\mathscr{C}_{\alpha}$ be the conjugacy class of all permutations in $\mathfrak{S}_{n}$ with $m_{1}(\alpha)$ cycles of length $1, m_{2}(\alpha)$ cycles of length $2, \ldots$ in their cycle decomposition. Let $\mathbf{K}_{\alpha}=\sum_{g \in \mathscr{C}_{\alpha}} g$, so $\mathbf{K}_{\alpha}$ is in the centre of $\mathbb{C} \mathfrak{S}_{n}$, the group algebra of $\mathfrak{\Xi}_{n}$. The set of all $\mathbf{K}_{\alpha}$ with $\alpha \vdash n$ is a basis of the centre, so $\left[\mathbf{K}_{\gamma}\right] \mathbf{K}_{\alpha} \mathbf{K}_{\beta}$, the coefficient of $\mathbf{K}_{\gamma}$ in $\mathbf{K}_{\alpha} \mathbf{K}_{\beta}$ is well defined. Since $\mathbb{C}_{n}$ is semi-simple, it has a basis $\left\{\mathbf{F}_{\alpha}: \alpha \vdash n\right\}$ of orthogonal idempotents defined by $\mathbf{F}_{\alpha}=\frac{f^{\alpha}}{n !} \sum_{\theta \vdash n} \chi_{\theta}^{\alpha} \mathbf{K}_{\theta}$, and, by orthogonality, $\mathbf{K}_{\alpha}=\frac{1}{n !} h^{\alpha} \sum_{\theta \vdash n} \chi_{\alpha}^{\theta} \mathbf{F}_{\theta}$. Thus $\left[\mathbf{K}_{\gamma}\right] \mathbf{K}_{\alpha} \mathbf{K}_{\beta}=\frac{1}{n !} h^{\alpha} h^{\beta} \sum_{\theta \vdash n} \chi_{\alpha}^{\theta} \chi_{\beta}^{\theta} \chi_{\gamma}^{\theta}$, so, from (7),

$$
R(\mathbf{x}, \mathbf{y}, z)=\sum_{n \geqq 0} \frac{z^{n}}{n !} \sum_{v, \phi \vdash 2 n} x_{v} y_{\phi} \frac{h^{\phi}}{h^{\left[2^{n}\right]}}\left[\mathbf{K}_{\phi}\right] \mathbf{K}_{v} \mathbf{K}_{\left[2^{n}\right]} .
$$

But $\left[\mathbf{K}_{\gamma}\right] \mathbf{K}_{\alpha} \mathbf{K}_{\beta}$ is identified combinatorially as the number of ways of expressing an arbitrary element $c \in \mathscr{C}_{\gamma}$ as $c=a b$ with $(a, b) \in \mathscr{C}_{\alpha} \times \mathscr{C}_{\beta}$. Thus

whence

$$
\left|\mathscr{C}_{\alpha} \mathscr{C}_{\beta} \cap \mathscr{C}_{\gamma}\right|=\frac{1}{n !} h^{\alpha} h^{\beta} h^{\gamma} \sum_{\theta \vdash n} \frac{1}{f^{\theta}} \chi_{\alpha}^{\theta} \chi_{\beta}^{\theta} \chi_{\gamma}^{\theta}
$$

$$
R(\mathbf{x}, \mathbf{y}, z)=\sum_{n \geqq 0} \frac{z^{n}}{n !} \sum_{v, \phi \vdash 2 n} x_{v} y_{\phi}\left|\mathscr{C}_{v} \varepsilon_{n} \cap \mathscr{C}_{\phi}\right|,
$$

where $\varepsilon_{n}$ is an arbitrary, but fixed, element of $\mathscr{C}_{\left[2^{n}\right]}$. This expresses $R(\mathbf{x}, \mathbf{y}, z)$ as a combinatorial sum, which will be used later.

\section{The 4- and Penner-Models}

The remaining part of this paper is concerned with the connexion between $Z_{N}(U)$ or $\mathscr{Z}_{N}(U, \beta)$ and the genus series for maps. The argument is independent of the integral representation of the genus series. Extensive use is made of enumerative properties of combinatorial structures. The main aspects that are used here are given below. The reader is referred to [11] for further details.

3.1. Enumerative Information. Let $\mathbb{Q}[[x]]$ be the ring of formal power series in the indeterminate $x$ with coefficients in $\mathbb{Q}$. Let $\mathbb{Q}[[x]]_{1}$ be the set of all such series with nonzero constant term, and let $\mathbb{Q}[[x]]_{0}$ be those with constant term 0 . 
Let $\mathscr{S}$ be a set of combinatorial configurations, and let $\mathbf{u}$ be a generic subobject of such configurations. A weight function is a function $\sigma: \mathscr{S} \rightarrow\{0,1,2, \ldots\}$ such that $\sigma(s)$ is the number of occurrences of the generic element $\mathbf{u}$ in $s \in \mathscr{S}$. A combinatorial problem is $(\mathscr{S}, \sigma)$. Let $\mathbf{O}=\left(1, x, x^{2}, \ldots\right)$, and $\mathbf{E}=\left(1, x / 1 !, x^{2} / 2 !, \ldots\right)$. These are bases of $\mathbb{Q}[[x]]$. The ordinary and exponential generating series are, respectively, $[(\mathscr{S}, \sigma)]_{\mathrm{o}}=\sum_{s \in \mathscr{S}} x^{\sigma(s)}$, and $[(\mathscr{S}, \sigma)]_{\mathbf{E}}=\sum_{s \in \mathscr{S}} x^{\sigma(s)} / \sigma(s)$ !, so the enumerative information, $|\{s \in \mathscr{S}: \sigma(s)=n\}|$, is recovered by taking $\left[x^{n}\right][(\mathscr{S}, \sigma)]$ o, or $\left[x^{n} / n !\right][(\mathscr{S}, \sigma)]_{\mathbf{E}}$.

\subsubsection{Combinatorial Operations on Sets}

1) Weight-Preserving Bijections: Let $(\mathscr{T}, \tau)$ be a combinatorial problem. If $\Omega: \mathscr{S} \stackrel{\sim}{\rightarrow}$ $\mathscr{T}$ is a bijection such that $\sigma=\tau \Omega$, then $\Omega$ is said to be weight-preserving. Since, in this case $[(\mathscr{S}, \sigma)]_{\mathbf{o}}=[(\mathscr{T}, \tau)]_{\mathbf{o}}$, and $[(\mathscr{S}, \sigma)]_{\mathbf{E}}=[(\mathscr{T}, \tau)]_{\mathbf{E}}$, we seek weightpreserving bijections whose domains and codomains are disjoint unions and products of sets. Although there is no effective algorithm for determining such bijections, the deletion of a canonical subconfiguration often leads to a decomposition, and this is the case for maps.

2) Products: Let $(\mathscr{A}, \alpha),(\mathscr{B}, \beta)$ and $(\mathscr{A} \times \mathscr{B}, \omega)$ be combinatorial problems such that $\omega((a, b))=\alpha(a)+\beta(b)$, and we write $\omega=\alpha \oplus \beta$. Then $[(\mathscr{A} \times \mathscr{B}, \alpha \oplus \beta)]_{\mathbf{o}}=$ $[(\mathscr{A}, \alpha)]_{\mathbf{o}} \cdot[(\mathscr{B}, \beta)]_{\mathbf{o}}$.

Let $\star$ be an operation on sets such that $[(\mathscr{A} \star \mathscr{B}, \alpha \oplus \beta)]_{\mathbf{E}}=[(\mathscr{A}, \alpha)]_{\mathbf{E}} \cdot[(\mathscr{B}, \beta)]_{\mathbf{E}}$. This operation may be realised as follows. Let $a \in \mathscr{A}$, where $a$ has the numbers $1, \ldots, \alpha(a)$ assigned to its subobjects $\mathbf{u}$. We say that $a$ is a labelled configuration, and $\mathscr{A}$ is a set of labelled configurations. Let $\mathscr{B}$ be a set of labelled configurations. Then $\mathscr{A} \star \mathscr{B}$ is a set of labelled configurations obtained from $(a, b) \in \mathscr{A} \times \mathscr{B}$ by uniquely relabelling the subobjects of $(a, b)$ with labels $1, \ldots, \alpha(a)+\beta(b)$, in all possible ways.

Let $\left[\mathbf{u}^{n}\right] \mathscr{S}$ denote the set of all elements in $\mathscr{S}$ with $n$ subobjects when the configurations are not labelled. Let $\left[\mathscr{N}_{n}\right] \mathscr{S}$ denote the set of all elements in $\mathscr{S}$ with $n$ subobjects when the configurations are labelled.

If $\mathscr{A} \stackrel{\sim}{\rightarrow} \mathscr{A}_{1} \times \cdots \times \mathscr{A}_{p}$ or $\mathscr{A} \stackrel{\sim}{\rightarrow} \mathscr{A}_{1} \star \cdots \star \mathscr{A}_{p}$ for a set of combinatorial configurations $\mathscr{A}$, then $\mathscr{A}_{1}, \ldots, \mathscr{A}_{p}$ are called the sets of primes of this combinatorial factorisation. Of course, $\mathscr{A}$ may admit more that one prime factorisation.

3) Composition of structures: For composition with $\times$, let $\mathcal{O}_{k}=(1, \ldots, k)$ and $\mathcal{O}=\left\{\varepsilon, \mathcal{O}_{1}, \mathcal{O}_{2}, \ldots\right\}$. Let $\mathcal{O}_{k} \circ \mathscr{B}=\mathscr{B} \times \cdots \times \mathscr{B},(k$ terms $)$, where there is no $b \in \mathscr{B}$ such that $\beta(b)=0$, and $\mathcal{O} \circ \mathscr{B}=\bigcup_{k \geqq 0}\left(\mathcal{O}_{k} \circ \mathscr{B}\right)$. Similarly, for composition with $\star$, let $\mathscr{N}_{k}=\{1,2, \ldots, k\}$, for $k \geqq 1$, and $\mathscr{U}=\left\{\varepsilon, \mathscr{N}_{1}, \mathscr{N}_{2}, \ldots\right\}$. Let $\mathscr{N}_{k} \odot \mathscr{A}=$ $\mathscr{A} \star \ldots \star \mathscr{A} / \mathfrak{S}_{k},(k$ terms $)$, under the natural action of $\mathfrak{S}_{k}$ on the component positions, where there is no $a \in \mathscr{A}$ such that $\alpha(a)=0$. Let $\mathscr{U} \odot \mathscr{A}=\bigcup_{k \geqq 0}\left(\mathscr{N}_{k} \odot \mathscr{A}\right)$.

Let $\mathscr{G}$ be the set of all labelled graphs and $\mathscr{K}$ the set of all connected labelled graphs. Then $\mathscr{G} \stackrel{\sim}{\rightarrow} \mathscr{U} \odot \mathscr{K}$, since a graph is uniquely decomposable into its connected components as primes. If $v(\mathbf{G})$ is the number of vertices in $\mathbf{G} \in \mathscr{G}$, and $G(x)=[(\mathscr{G}, v)]_{\mathbf{E}}$ and $K(x)=[(\mathscr{K}, v)]_{\mathbf{E}}$, then

$$
G(x)=\exp K(x), \quad \text { and } \quad K(x)=\log G(x) .
$$


4) The Derivative of a Set: Let the u-derivative $(\mathbf{u} \partial / \partial \mathbf{u}) \mathscr{S}$ of $\mathscr{S}$ denote the set of all configurations obtained from $\mathscr{S}$ by distinguishing a unique subobject in each $s \in \mathscr{S}$ in every possible way. Then $[(\mathbf{u} \partial \mathscr{S} / \partial \mathbf{u}, \sigma)]_{\mathbf{o}}=x \partial[(\mathscr{S}, \sigma)]_{\mathbf{o}} / \partial x$, and $[(\mathbf{u} \partial \mathscr{S} / \partial \mathbf{u}, \sigma)]_{\mathbf{E}}=x \partial[(\mathscr{S}, \sigma)]_{\mathbf{E}} / \partial x$. Rooting a map involves distinguishing a subobject, in this case an edge.

Let $\mathscr{S}$ be the set of all labelled configurations with generic subobject $\mathbf{u}$. Then $\partial \mathscr{S} / \partial \mathbf{u}$ is the set of all elements obtained by deleting a canonical subobject from each element of $\mathscr{S}$. For example, $\mathbf{u} \partial \mathscr{S} / \partial \mathbf{u} \stackrel{\sim}{\rightarrow}\{\mathbf{u}\} \star(\partial \mathscr{S} / \partial \mathbf{u})$, since the object which is deleted is then reintroduced, distinguished, and all subobjects are relabelled in all possible ways. By adjusting $\{\mathbf{u}\}$ we can therefore distinguish a subobject in more than one way.

5) Superposition: Let $\mathscr{A}, \mathscr{B}$ be sets of combinatorial configurations on the same set of labelled subobjects. For $a \in \mathscr{A}$ and $b \in \mathscr{B}$, let $\omega(a), \omega(b)$ be the numbers of subobjects in $a$ and $b$, respectively. When $\omega(a)=\omega(b)$, then $a \diamond b$ denotes the configuration obtained from $a$ and $b$ by identifying the two subobjects labelled $i$, one from each, for $i=1, \ldots, \omega(a)$; otherwise, $a \diamond b$ is undefined. This operation is called superposition of $a$ and $b$. Let $\mathscr{A} \diamond \mathscr{B}=\{a \diamond b:(a, b) \in \mathscr{A} \times \mathscr{B}, \omega(a)=\omega(b)\}$. Then $[(\mathscr{A} \diamond \mathscr{B}, \omega)]_{\mathbf{E}}=[(\mathscr{A}, \omega)]_{\mathbf{E}} \diamond[(\mathscr{B}, \omega)]_{\mathbf{E}}$, where $\left(\sum_{i \geqq 0} a_{i} x^{i} / i !\right) \diamond\left(\sum_{i \geqq 0} b_{i} x^{i} / i !\right)$ $=\left(\sum_{i \geqq 0} a_{i} b_{i} x^{i} / i\right.$ ! $)$, the Hadamard product of the two series in the basis $\mathbf{E}$ of $\mathbb{Q}[[x]]$.

For example, let $\mathscr{F}$ be the set of all 1-matchings, labelled graphs whose vertices have degree one, only. Necessarily, such graphs have an even number of vertices. Since $\mathscr{F} \stackrel{\sim}{\rightarrow} \mathscr{U} \odot(\{1,2\})$, the generating series for $\mathscr{F}$ is $\exp \left(\frac{1}{2} x^{2}\right)$. Then

$$
\mathscr{F} \diamond \mathscr{F} \stackrel{\sim}{\rightarrow} \mathscr{U} \odot \mathscr{E},
$$

where $\mathscr{E}$ is the set of all cycles of even length with edges of alternating colours. This is because edges of one colour come from the left $\mathscr{F}$ of the superposition and edges of the other colour come from the right $\mathscr{F}$. Since these cycles are bicoloured, they are in 1-1 correspondence with even length cycles in permutations.

6) The Laplace Transform: The final operation concerns the attachment of labels to the subobjects of an unlabelled configuration. Let $s \in \mathscr{S}$, and suppose that the subobjects, $\mathbf{u}$, of $s$ can be canonically labelled from 1 to $n$. Let $f$ be a bijection on $1, \ldots, n$. Let $\mathscr{L}[\mathscr{S}]$ denote the set of all such $(s, f)$. Then $\mathscr{S}$ is said to be u-uniform if $(s, f)$ and $\left(s^{\prime}, f^{\prime}\right)$ are distinguishable if and only if they are not equal. If $\mathscr{S}$ is u-uniform then $[(\mathscr{S}, \sigma)]_{\mathbf{o}}=[(\mathscr{L}[\mathscr{S}], \sigma)]_{\mathbf{E}}$. This operation corresponds to the formal Laplace transform.

\subsubsection{The Inverse Problem and Refinement}

1) The Inverse Problem: For a given series $C(x)$, we seek a set $\mathscr{C}$, a weight function $\gamma$ and a choice $\mathbf{O}, \mathbf{E}$ of basis for $\mathbb{Q}[[x]]$, such that $[(\mathscr{C}, \gamma)]_{\mathbf{0}}=C(x)$ or $[(\mathscr{C}, \gamma)]_{\mathrm{E}}=C(x)$ by constructing $\mathscr{C}$ from atomic sets under the above operations and the action of an appropriate weight-preserving bijection $\Omega$. If $C(x)$ is constructed as the solution of a functional equation, or if it is a finitary combination of sums, products and compositions then the above formalism gives some information about an elementwise action for $\Omega$, and a configuration and a weight function. Since the 
mapping from sets to generating series is not injective, the resulting configuration is not unique.

2) Refinement of the Weight Function: Let $\omega_{1}, \omega_{2}, \ldots: \mathscr{S} \rightarrow \mathscr{N}$ and let $\omega_{1} \otimes \omega_{2} \otimes$ $\cdots: s \mapsto\left(\omega_{1}(s), \omega_{2}(s), \ldots\right)$. For $\mathbf{B}_{1}, \mathbf{B}_{2}, \ldots \in\{\mathbf{O}, \mathbf{E}\}$, let $\left[\left(\{s\}, \omega_{1} \otimes \omega_{2} \otimes \cdots\right)\right]_{\mathbf{B}_{1}, \mathbf{B}_{2}, \ldots}$ $=t_{1} t_{2} \cdots$, where $t_{j}=x_{j}^{\omega_{j}(s)}$ if $\mathbf{B}_{j}=\mathbf{O}$, and $t_{j}=x_{j}^{\omega_{j}(s)} /\left(\omega_{j}(s)\right)$ ! with $\omega_{j}(s)$ being the number of subobjects of $s$ if $\mathbf{B}_{j}=\mathbf{E}$. If the indeterminates are not specified, it is to be assumed that one is assigned to each weight function, and that they are independent. We define the operation $\otimes$ on $\left[\left(\mathscr{A}, \omega_{1}\right)\right]_{\mathbf{B}_{1}}$ and $\left[\left(\mathscr{A}, \omega_{1}\right)\right]_{\mathbf{B}_{2}}$ by $\left[\left(\mathscr{A}, \omega_{1}\right)\right]_{\mathbf{B}_{1}} \otimes\left[\left(\mathscr{A}, \omega_{2}\right)\right]_{\mathbf{B}_{2}}=\left[\left(\mathscr{A}, \omega_{1} \otimes \omega_{2}\right)\right]_{\mathbf{B}_{1}, \mathbf{B}_{2}}$. This therefore corresponds to a refinement of the weight for $\mathscr{A}$ from $\omega_{1}$ or $\omega_{2}$, to $\omega_{1}$ and $\omega_{2}$, simultaneously, and is a tensor product of generating series for a fixed set, with respect to different weight functions. The product lemma can be generalised as follows. Let $\alpha_{1}, \alpha_{2}: \mathscr{A} \rightarrow \mathcal{N}, \beta_{1}, \beta_{2}: \mathscr{B} \rightarrow \mathscr{N}, \omega_{1}=\alpha_{1} \oplus \beta_{1}, \omega_{2}=\alpha_{2} \oplus \beta_{2}$. Then $[(\mathscr{A} \times$ $\left.\left.\mathscr{B}, \omega_{1} \otimes \omega_{2}\right)\right]_{\mathbf{B}_{1}, \mathbf{B}_{2}}=\left[\left(\mathscr{A}, \alpha_{1} \otimes \alpha_{2}\right)\right]_{\mathbf{B}_{1}, \mathbf{B}_{2}}\left[\left(\mathscr{A}, \beta_{1} \otimes \beta_{2}\right)\right]_{\mathbf{B}_{1}, \mathbf{B}_{2}}$, where $\omega_{1} \otimes \omega_{2}=\alpha_{1} \otimes$ $\alpha_{2} \oplus \beta_{1} \otimes \beta_{2}$. We may suppress the bases, on the understanding that the same ordered list is attached to each quantity, and that for $\mathbf{O}$ the product is $\times$ and for $\mathbf{E}$ the product is $\star$. Letting $\alpha=\alpha_{1} \otimes \alpha_{2}$ and $\beta=\beta_{1} \otimes \beta_{2}$, we have $[(\mathscr{A} \times \mathscr{B}, \alpha \otimes \beta)]=$ $[(\mathscr{A}, \alpha)][(\mathscr{B}, \beta)]$. The terms ordinary and exponential are confined to the univariate series of these types in the tensor product.

3) Reduction to the Univariate Case: Let $\omega_{1}, \omega_{2}, \ldots$ be weight functions for $\mathscr{A}$, and let $\omega=\omega_{1}+\omega_{2}+\cdots$. A bijection that preserves $\omega$ may also preserve $\omega_{1} \otimes$ $\omega_{2} \otimes \cdots$. For example, if the number of vertices in a map is preserved under a bijection, then the number of vertices of each degree is usually preserved also. There is a natural isomorphism $\mathbb{Q}\left[\left[x_{1}, \ldots, x_{n}\right]\right] \rightarrow \mathbb{Q}\left[\left[x_{1}, \ldots, x_{j-1}, x_{j+1}, \ldots, x_{n}\right]\right]\left[\left[x_{j}\right]\right]$. This permits attention to be focussed upon a particular indeterminate and the generating series is then univariate, and so ordinary or exponential, the coefficient ring having been changed under this isomorphism. It is particularly useful for the genus series, with constructions affecting edges, where it is clear that vertex degrees and face degrees are preserved.

3.1.3. Two Examples. A planted plane tree is a tree, embedded in the plane, with a monovalent root vertex. Let $\mathscr{T}$ be the set of all planted plane trees. On deletion of the (unique) vertex adjacent to the root of $t \in \mathscr{T}, t$ decomposes into a linearly ordered set of subtrees of $t$, each in $\mathscr{T}$, induced by the orientation of the surface. Thus, $\mathscr{T} \stackrel{\sim}{\rightarrow}\{\mathbf{s}\} \times(\mathcal{O} \circ \mathscr{T})$, where $\{\mathbf{s}\}$ is a generic nonroot vertex. Similarly, let $\mathscr{A}$ be the set of all labelled rooted trees. Then $\mathscr{A} \stackrel{\sim}{\rightarrow}\{\mathbf{u}\} \star(\mathscr{U} \odot \mathscr{A})$, where $\mathbf{u}$ is the generic vertex.

As an example of the combinatorial operations and the checking of weight preservation we prove Lagrange's theorem and MacMahon's theorem. The former is used here in specialisation of the genus series. Let $\mathscr{N}_{n}=\{1, \ldots, n\}$, where $n$ is a positive integer.

1) Lagrange's Theorem: Lagrange's implicit function theorem (see, for example, [11]), adapted to the ring of formal power series, states that if $\mathbb{K}$ is a ring with unity and $\phi \in \mathbb{K}[[\lambda]]_{1}$, then there exists a unique series $w \in \mathbb{K}[[t]]_{0}$ such that

$$
w=t \phi(w)
$$


and, for $F \in \mathbb{K}[[\lambda]]$,

$$
\begin{aligned}
{\left[t^{n}\right] F(w) } & =\frac{1}{n}\left[\lambda^{n-1}\right] F^{\prime}(\lambda) \phi^{n}(\lambda), \quad \text { where } n>0, \\
\sum_{n \geqq 0} c_{n} t^{n} & =\frac{F(w)}{1-t \phi^{\prime}(w)}, \quad \text { where } c_{n}=\left[\lambda^{n}\right] F(\lambda) \phi^{n}(\lambda) .
\end{aligned}
$$

This can be proved combinatorially by a chain of bijections mapping a rooted labelled tree into a function. Each bijection can be checked by supplying the elementwise actions of natural combinatorial mappings, each of which is classical. We regard the generating series as a univariate exponential generating series in the indeterminate $x$ marking nonroot vertices of a tree or elements of the domain of the corresponding function, and use the tensor product construction to record degree sequence and pre-image size sequence, having checked weight preservation.

Let $\mathscr{P}$ be the set of all permutations on $\mathscr{N}_{n}$, for all $n \geqq 0$, and let $\mathscr{C} \subset \mathscr{P}$ be the set of those with exactly one cycle. Let $\mathbf{u}$ denote the subobject consisting of a generic vertex. Let $\mathscr{A}$ denote the set of rooted labelled trees, with only the non-root vertices labelled. Thus $\mathscr{A}=\bigcup_{k} \mathscr{A}_{k}$, where $\mathscr{A}_{k}$ is the set of all rooted trees in which only the nonroot vertices are labelled, and with root degree $k$. The label 0 is assigned to the root of trees in $\mathscr{A}_{k}$. Direct all edges of such trees towards the root vertex.

Let $t \in \mathscr{A}_{k}$ have $n$ nonroot vertices. Delete the edges of the unique path $\pi$ in $t$ from 0 to $n$. Under this operation, the in-degree of 0 is $k-1$, the in-degree of each interior vertex of $\pi$ is decreased by 1 , and the in-degrees of $n$ and the remaining vertices are unchanged. On deleting $\pi, t$ decomposes into an ordered forest of trees in $\mathscr{A}$, so $\left[\mathscr{N}_{n}\right] \mathscr{A}_{k} \stackrel{\sim}{\rightarrow}\left[\mathscr{N}_{n-1}\right]\left\{\mathscr{A}_{k-1} \star(\mathscr{P} \odot \widehat{A}) \star \mathscr{A}\right\}$, where $\widehat{\mathscr{A}}$ is the set of all rooted labelled trees. Let $t \mapsto(a, s, b)$ under this bijection. The label 0 is assigned to the root of $a$, and the label $n$ is assigned to the root of $b$. From the above observations, the in-degree sequence is not preserved by this bijection. Indeed, to preserve this sequence it is necessary to add one to each of the root-degrees of trees in $\mathscr{P} \odot \widehat{A}$. To do this, note that by the cycle decomposition for permutations, $\mathscr{P} \odot \widehat{\mathscr{A}} \stackrel{\sim}{\rightarrow}(\mathscr{U} \odot \mathscr{C}) \odot \widehat{\mathscr{A}} \stackrel{\sim}{\rightarrow} \mathscr{U} \odot(\mathscr{C} \odot \widehat{\mathscr{A}})$, since $\odot$ is a composition. The elements of $\mathscr{U} \odot(\mathscr{C} \odot \widehat{A})$ are labelled graphs whose components are unicursal (connected graphs with one cycle). In each component, direct all tree edges towards the cycle, and direct the cycle. Let $s \mapsto s^{\prime}$ under this bijection. Then the root vertices of trees in $s$ are the interior vertices of $\pi$, and are also the vertices on cycles in $s^{\prime}$. The in-degrees of such vertices in $s^{\prime}$ are one greater than the in-degrees of the vertices on cycles in $s^{\prime}$. The in-degrees of the remaining vertices in $s^{\prime}$ are equal to the in-degrees of the remaining vertices in $s$. It follows that

$$
\left[\mathscr{N}_{n}\right] \mathscr{A}_{k} \stackrel{\sim}{\rightarrow}\left[\mathscr{N}_{n-1}\right]\left(\mathscr{A}_{k-1} \star\{\mathscr{U} \odot(\mathscr{C} \odot \widehat{\mathscr{A}})\} \star \mathscr{A}\right)
$$

is in-degree preserving. But $\mathscr{U} \odot(\mathscr{C} \odot \widehat{\mathscr{A}})$ is the set of all functional digraphs, since an edge directed from vertex $i$ to vertex $j$ specifies the action $i \mapsto j$ of a function. Let $\mathbf{s}$ denote the generic subobject of the domain of such a function. Then $\mathscr{U} \odot(\mathscr{C} \odot \mathscr{A}) \stackrel{\sim}{\rightarrow} \bigcup_{m \geqq 0} \mathscr{N}_{m}^{\mathscr{N}_{m}}$, whence $\left[\mathscr{N}_{n}\right] \mathscr{A}_{k} \stackrel{\sim}{\rightarrow} \mathscr{W}_{n}$ where, $\mathscr{W}_{n}=$ $\left\{f \in\{0,1, \ldots, n\}^{\mathscr{N}_{n-1}}:\left|f^{-1}(0)\right|=k-1\right\}$. Let $t \mapsto f$ under this bijection. Then the in-degree sequence of $t$ is equal to the pre-image size sequence of $f$. But $\mathscr{N}_{n}^{\mathscr{N}_{n-1}} \stackrel{\sim}{\rightarrow}\left[\mathscr{N}_{n-1}\right]\left\{\mathcal{O}_{n} \odot \mathscr{U}\right\}$, since each such function is uniquely encoded by an 
ordered partition of $\mathscr{N}_{n-1}$ with $n$ blocks, the $j^{\text {th }}$ block being the pre-image of $j$, for $j=1, \ldots, n$. It follows that $\mathscr{W}_{n} \stackrel{\sim}{\rightarrow}\left[\mathscr{N}_{n-1}\right]\left\{\mathscr{N}_{k-1} \star\left(\mathcal{O}_{n} \odot \mathscr{U}\right)\right\}$. We conclude that $\left[\mathscr{N}_{n}\right] \mathscr{A}_{k} \stackrel{\sim}{\rightarrow}\left[\mathscr{N}_{n-1}\right]\left\{\mathscr{N}_{k-1} \star\left(\mathcal{O}_{n} \odot \mathscr{U}\right)\right\}$. Moreover, if $t \mapsto q$ under this bijection, then the in-degree sequence of $t$ is the block size sequence of the partition $q$, so the bijection is weight-preserving. Now $\mathscr{N}_{k-1} \stackrel{\sim}{\rightarrow} \partial \mathscr{N}_{k} / \partial \mathbf{s}$, so, summing over $k$

$$
\left[\mathscr{N}_{n}\right](\mathscr{U} \odot \widehat{\mathscr{A}}) \stackrel{\sim}{\rightarrow}\left[\mathscr{N}_{n-1}\right]\left\{\frac{\partial \mathscr{U}}{\partial \mathbf{s}} \star\left(\mathcal{O}_{n} \odot \mathscr{U}\right)\right\} .
$$

If $t \mapsto(\partial a / \partial \mathbf{s}, b)$ under this bijection, then the size of $a$ is the root-degree of $t$. This bijection therefore preserves the degree of the root and the in-degree sequence of $t$.

Let $a_{j}$ be an indeterminate marking nonroot vertices in $t$ with in-degree $j$ and pre-images under $f$ of size $j$, for $j \geqq 0$. Let $\phi(x)=a_{0}+a_{1} x+a_{2} x^{2} / 2 !+\cdots$. Let $b_{j}$ be an indeterminate marking the root degree in $t$ with in-degree $j$ and the size $j$ of the preimage of 0 in $f$. Let $F(x)=b_{1} x+b_{2} x^{2} / 2$ ! $+\cdots$. Then, from (43) $\left[x^{n}\right] F(T)=\frac{1}{n}\left[x^{n-1}\right] F^{\prime}(x) \phi^{n}(x)$, where $T(x)$ is the exponential generating series for $\widehat{\mathscr{A}}$ with respect to nonroot vertices. Finally, $\widehat{\mathscr{A}} \stackrel{\sim}{\rightarrow}\{\mathbf{u}\} \star(\mathscr{U} \odot \widehat{\mathscr{A}})$, so $T(x)$ satisfies the functional equation $T=x \phi(T)$. In other notation, this establishes (41) and (40). Finally, (42) can be proved immediately from (41) by differentiating $F(w)$ with respect to $t$.

2) MacMahon's Master Theorem: Let $\mathbf{A}$ be an $n \times n$ matrix, $k_{1}, \ldots, k_{r}$ be nonnegative integers, $\mathbf{k}=\left(k_{1}, \ldots, k_{n}\right)$, and $\mathbf{X}=\operatorname{diag}\left(x_{1}, \ldots, x_{n}\right)$. Then

$$
\left[\mathbf{x}^{\mathbf{k}}\right] \prod_{i=1}^{n}\left(a_{i, 1} x_{1}+\cdots+a_{i, n} x_{n}\right)^{k_{l}}=\left[\mathbf{x}^{\mathbf{k}}\right] \operatorname{det}(\mathbf{I}-\mathbf{X A})^{-1} .
$$

Let $f \in \mathscr{N}_{n}^{\mathscr{N}_{n}}$, and let $\omega(f)=\prod_{i=1}^{n} a_{i, f(i)}$. Let $\mathscr{B}_{n}$ be the set of all bijections in $\mathscr{N}_{n}^{\mathscr{N}_{n}}$. We count $\mathscr{B}_{n}$ with respect to $\omega$ by encoding the action of an element of $\mathscr{B}_{n}$ in two different ways. First, $\mathscr{B}_{n} \stackrel{\sim}{\rightarrow}\left[\mathscr{N}_{n}\right] \mathscr{P}$. If $f \mapsto \pi$ under this bijection, let $\sigma(\pi)=\prod_{i=1}^{n} a_{i, \pi(i)}$. Then this bijection is $\omega$-preserving. Let $\mathfrak{C}_{k}$ denote the cyclic group on $k$ symbols. Now $\left\{\left[\mathscr{N}_{k}\right]\left(\mathscr{O}_{k} \circ \mathscr{N}\right)\right\} / \mathfrak{C}_{k}$ is the set of all circular strings on $\mathscr{N}_{k}$ with exactly one occurrence of each of the symbols in $\mathscr{N}_{k}$. If $s=i_{1} i_{2} \ldots i_{k}$ is in this set, let $\sigma(s)=a_{i_{1}, i_{2}} a_{i_{2}, i_{3}} \ldots a_{i_{k}, i_{1}}$. By cycle decomposition $\left[\mathscr{N}_{n}\right] \mathscr{P} \stackrel{\sim}{\rightarrow}\left[\mathscr{N}_{n}\right]\left(\mathscr{U} \circ\left\{\bigcup_{k \geqq 1}\left\{\left[\mathscr{N}_{k}\right]\left(\mathcal{O}_{k} \circ \mathscr{N}\right)\right\} / \mathscr{C}_{k}\right\}\right)$. If $\pi \mapsto s$ then $\omega(\pi)=\sigma(s)$, so the bijection is weight-preserving. Second, $\mathscr{B}_{n} \stackrel{\sim}{\rightarrow}\left[\mathscr{N}_{n}\right]\left(\mathcal{O}_{n} \circ \mathscr{N}_{n}\right)$, since $\mathcal{O}_{n} \circ \mathscr{N}_{n}$ is an ordered set of nonempty blocks, encoding the pre-images of a surjection, and $\left[\mathscr{N}_{n}\right]$ ensures that the function is injective. This is also weight-preserving. Thus $\left[\mathscr{N}_{n}\right]\left(\mathcal{O}_{n} \circ \mathscr{N}_{n}\right) \stackrel{\sim}{\rightarrow}\left[\mathscr{N}_{n}\right]\left(\mathscr{U} \circ\left\{\bigcup_{k \geqq 1}\left\{\left[\mathscr{N}_{k}\right]\left(\mathcal{O}_{k} \circ \mathscr{N}\right)\right\} / \mathscr{C}_{k}\right\}\right)$ on equating these. Thus $[\mathbf{x}] \prod_{i=1}^{n} \sum_{j=1}^{n} a_{i, j} x_{j}=[\mathbf{x}] \exp \sum_{k \geqq 1} \frac{1}{k} \operatorname{trace}\left(\mathbf{A}^{k}\right)=[\mathbf{x}] \operatorname{det}(\mathbf{I}-\mathbf{X} \mathbf{A})^{-1}$, by a result of Jacobi. The result follows from this, under the transformation $a_{i, j} \mapsto a_{i, j} \mathbf{J}_{k_{l}}, k_{j}$, for $i, j=1, \ldots, n$, where $\mathbf{J}_{k_{i}, k_{j}}$ is the $k_{i} \times k_{j}$ matrix of ones.

3.2. Rotation Systems. Our purpose now is to obtain the relationship between $R(\mathbf{x}, \mathbf{y}, z)$, as a combinatorial sum, and the genus series for rooted maps. The notation that assists with this is given in Sect. 1.4. Each vertex $v$ of $G$ is represented as a subset of the directed edge set, so $e^{+}$is in the subset if and only if $v$ is the origin 
of $e$, and $e^{-}$is in the subset if and only if $v$ is the terminus of $e$. These subsets partition the directed edge set. For each vertex $v$ of $G$, we specify a cyclic list of the directed edges encountered in traversing the boundary of a small disc, centred at $v$, in the sense specified by the orientation of $\Sigma$. We shall fix this sense to be anticlockwise. This partition of the directed edge set into cyclic lists is called a rotation system, and it represents a permutation on the set $\left\{1^{+}, 1^{-}, \ldots, n^{+}, n^{-}\right\}$ of edge-ends. By a generalisation of a theorem ascribed to Schoenfliess [17] it is known that, for every embedding of $G$ in $\Sigma, G$ is contained in the 1-skeleton of a triangulation of this surface.

Every rotation system for a connected graph $G$ induces, up to equivalence of embeddings, a unique embedding of $G$ in $\Sigma$. However, not every permutation $v \in \Xi_{2 n}$ corresponds to a rotation system for an embedding of a graph with $n$ edges. If $v$ and $\varepsilon_{n}$ do not generate $\mathfrak{S}_{2 n}$ then the associated graph $G$ is not connected, and we say that the rotation system is not transitive. Thus every embedding of $G$ in $\Sigma$ induces a unique transitive rotation system. This one-to-one correspondence, called the embedding theorem, was given originally in dual form by Heffter [16] and has been generalised to graphs with loops and multiple edges by Gross and Alpert [14]. If $v$ is the rotation system of a graph $G$ on $n$ edges, and $\varepsilon_{n}=\left(1^{+} 1^{-}\right)\left(2^{+} 2^{-}\right) \cdots\left(n^{+} n^{-}\right)$ is the fixed permutation describing, in its cycles, the edges of $G$, then the cycles of $v \varepsilon_{n}$ list the directed edges encountered in traversing the boundary of the faces of the embedding in a direction consistent with the orientation of $\Sigma$. It follows from (37), with edges as labelled subobjects, that $R(\mathbf{x}, \mathbf{y}, z)$ is the generating series for rotation systems, with $x_{i}$ marking cycles of length $i$ in $v$ and $y_{j}$ marking cycles of length $j$ in $v \varepsilon_{n}$. Each permutation in $\mathfrak{\Xi}_{2 n}$ is a rotation system for an unordered collection of graph embeddings, and the cycles of $v \varepsilon_{n}$ describe the faces of the embeddings of the unordered collection.

Let $\mathscr{R}$ be the set of all rotation systems and let $\mathscr{T}$ be the subset of these which are transitive. Then

$$
\mathscr{U} \odot \mathscr{T} \stackrel{\sim}{\rightarrow} \mathscr{R} .
$$

Let $\mathscr{M}$ be the set of all rooted maps. Then $\mathscr{M}$ is e-uniform, where e denotes the generic edge as a subobject, since there is a unique root-preserving automorphism of the map. Now $\mathscr{M} \circ\{+,-\}$ is the set of all rooted maps with a "direction," specified by + or - , assigned to each edge, independent of the rooting. Then $\mathscr{L}[\mathscr{M} \circ\{+,-\}]$ is the set of all edge labelled rooted maps with directed edges.

On the other hand, rooting a map corresponds to picking one of the edge-end symbols. Since $\mathscr{T}$ is the set of all transitive rotation systems, the generic subobjects $\mathbf{S}$ are canonically paired edge-end symbols, as specified by $\varepsilon_{n}$. Thus $\{+,-\} \star$ $\frac{\partial \mathscr{T}}{\partial \mathbf{s}}$ is the set of all configurations obtained by distinguishing an edge-end, so by the embedding theorem,

$$
\mathscr{L}[\mathscr{M} \circ\{+,-\}] \stackrel{\sim}{\rightarrow}\{+,-\} \star \partial \mathscr{T} / \partial \mathbf{s} .
$$

Since the cycle-types of $v$ and $v \varepsilon_{n}$ are preserved, then from (44), $R(\mathbf{x}, \mathbf{y}, z)=$ $\exp [(\mathscr{T}, \omega)]_{\mathbf{E}}$, where $\omega(t)$ is the number of subobjects $\mathbf{s}$ in $t \in \mathscr{T}$, marked by $z$. Then from (45), $[(\mathscr{L}[\mathscr{M} \circ\{+,-\}], \lambda)]_{\mathbf{E}}=2 z \partial[(\mathscr{T}, \omega)]_{\mathbf{E}} / \partial z$, where $\lambda(m)$ is the number of edges in $m \in \mathscr{M}$. But $M(\mathbf{x}, \mathbf{y}, z)=[(\mathscr{M}, \lambda)]_{\mathbf{o}}$, the genus series for rooted maps. Then, using the Laplace transform,

$$
2 z \frac{\partial}{\partial z} \log R(\mathbf{x}, \mathbf{y}, z)=[(\mathscr{M} \circ\{+,-\}, \omega)]_{\mathbf{o}}=M(\mathbf{x}, \mathbf{y}, 2 z),
$$


since (45) also preserves vertex- and face-degree, so (8) of Theorem B follows with $z \mapsto \frac{1}{2} z$.

The symmetry of $R(\mathbf{x}, \mathbf{y}, z)$, which has been observed earlier, appears in $M(\mathbf{x}, \mathbf{y}, z)$ as a consequence of duality.

With the aid of (8), the relationship of Theorem A can be translated into a relationship between species of maps. Then from (8), $M_{4}(x, y, z)=2 z \frac{\partial}{\partial z} \log R_{4}(x, y$, $\left.\frac{1}{2} z\right)$, and $M_{\mathcal{N}}(x, y, z)=2 z \frac{\partial}{\partial z} \log R_{\mathscr{N}}\left(x, y, \frac{1}{2} z\right)$. Now $z \partial / \partial z=2\left(2 z^{2} y\right) \partial / \partial\left(2 z^{2} y\right)$, so Theorem $\mathrm{C}$ follows from Theorem $\mathrm{A}$.

Theorem $\mathrm{C}$ expresses the number of vertex 4-regular maps of given genus $g$ in terms of maps (with no conditions on vertex degree) on surfaces of genus $g$ and lower.

The genus series enables us, at least in principle, to investigate classes of maps which can be defined by restrictions applied to vertex degrees and face degrees. Equations (3) and (7) give two ways of determining the genus series, one through integration, and the other through the determination of character sums.

3.3. A Combinatorial Construction for Feynman Maps. It has been observed in Sect. 1.2 that it is essential in applications to physics to consider maps with no vertices of degree one or two. A smooth map is a map with no monovalent vertices. A homeomorphically irreducible map is a map with no bivalent vertices. Let $M_{\overline{1}}(x, y, z), M_{\overline{2}}(x, y, z), M_{\overline{1}}(x, y, z)$, be the genus series for smooth maps, homeomorphically irreducible maps, and homeomorphically irreducible smooth maps, respectively. The relationship given in Theorem $\mathrm{C}$ cannot hold in such a simple form for smooth maps, but it is expected that a relationship of some sort remains. It is stated in Theorem $\mathrm{D}$, and is now derived combinatorially. We do this in two steps, first by accounting for monovalent vertices and then accounting for bivalent vertices.

3.3.1. Suppression of Monovalent Vertices. Let $m$ be a rooted map. By recursively deleting monovalent vertices of $m$, the latter is associated with a unique rooted smooth map $s$. It remains to be shown that $m$ can be reconstructed from $s$ by the attachment of appropriate trees.

A corner of a map is a mutually incident vertex, pair of edges, and face. Now assume that $m$ has at least two faces, so the corresponding smooth map $s$ has at least two corners. Then $m$ is reconstructed from $s$ by associating a planted plane tree with each corner. The corners are partitioned into ordered pairs as follows. Orient each face of $s$ in a sense consistent with the orientation of the surface. Assign a direction arbitrarily to each edge. Each edge separates a pair of faces, which can be designated $\mathbf{L}$ and $\mathbf{R}$ such that $\mathbf{L}$ is on the left side of the edge when looking in the direction of the edge. On the $\mathbf{L}$ side of an edge, the symbols $\mathbf{T L}$ and $\mathbf{H L}$ are attached at different ends so that the direction from $\mathbf{T}$ to $\mathbf{H}$ is consistent with the orientation of the face containing the L. Symbols TR and HR are similarly attached to the $\mathbf{R}$ side of the same edge. Then each edge defines an ordered pair (HR, HL). The set of all such ordered pairs is a covering of the corners of $s$.

Now consider an (HR, HL) pair corresponding to a nonroot edge. These correspond to an ordered pair of planted plane trees $\left(t_{1}, t_{2}\right)$. These are the trees that were removed under recursive deletion of monovalent vertices. A planted plane tree is attached to a corner by deleting the root vertex and incident edge of the tree, and by identifying the vertex of the tree, that was adjacent to the root, with the vertex 
of the corner so that the edges of the corner do not separate edges of the tree. This can be done in a unique way. The pair $\left(t_{1}, t_{2}\right)$ can be encoded as a single planted plane tree $t$ as follows. Identify the root vertex of $t_{2}$ with the vertex adjacent to the root $r_{1}$ of $t_{1}$, and the root of $t_{1}$ with the vertex adjacent to the root $r_{2}$ of $t_{2}$. Identify the root edges of $t_{1}$ and $t_{2}$, and direct it from $t_{1}$ to $t_{2}$. Attach an edge $f$ from a new vertex $r$ to $r_{2}$ so that, in counterclockwise circulation of $r_{2}$, starting from the edge $f$, the edge $\left\{r_{1}, r_{2}\right\}$ is encountered next, and then the edges of $t_{1}$. Root the tree at $r$ to obtain the planted plane tree $t$. The edge $\left\{r_{1}, r_{2}\right\}$ of $t$ is the edge of $s$ associated with a (HR, HL) pair. Let $\hat{\mathbf{e}}$ be the generic nonroot edge as a subobject, and let 2 denote the set of all planted plane trees on at least one nonroot edge (therefore at least two nonroot vertices). Then the above action constructs $\mathscr{M}_{\{\overline{1}\}} \circ \mathcal{Q}$. Now carry out the same construction for the root edge of $s$ but, in addition, distinguish an edge of the tree $t$, and direct it away from the root vertex. This is possible since every edge of a tree is on a path from a monovalent vertex to the root. This is designated as the root edge of the map $m$. This action constructs $\hat{\mathbf{e}} \partial \mathscr{Q} / \partial \hat{\mathbf{e}}$. It follows that

$$
\mathscr{M}-\mathscr{Q} \stackrel{\sim}{\rightarrow}\left(\frac{\hat{\mathbf{e}} \partial}{\partial \hat{\mathbf{e}}} \mathscr{Q}\right) \times\left(\mathscr{M}_{\{\overline{1}\}} \circ \mathscr{Q}\right) .
$$

If $q \in \mathscr{Q}$, the weight of $q$ is $\omega(q)=(i-2, k)$, where $q$ has $i$ nonroot vertices and $k$ nonroot edges. Then this bijection is weight preserving. Let $T(x, z)$ be the generating series for the set $\mathscr{T}$ of planted plane trees with respect to nonroot vertices and nonroot edges. Let $\mathbf{s}$ denote the generic nonroot vertex. Then $\mathscr{T} \stackrel{\sim}{\rightarrow}\{\mathbf{s}\} \times$ $(\mathcal{O} \circ \mathscr{T})$. Then $T=x /(1-z T)$. Let $Q(x, z)$ be the generating series for $\mathscr{Q}$ with respect to $\omega$. Let $t$ be the tree in $\mathscr{T}$ with one edge. Then $\mathscr{Q} \stackrel{\sim}{\rightarrow} \mathscr{T}-\{t\}$, so $Q=(T-x) / x^{2}$ and $Q$ satisfies the functional equation

$$
Q=z(1+x Q)^{2} \text {. }
$$

The generating series for $\mathscr{M}-\mathscr{Q}$ is $M(x, y, z)-x^{2} y Q$, since trees have only one face and two nonroot vertices have not been counted. The genus series for smooth maps with respect to nonroot vertices is $z^{-1} M_{\overline{1}}(x, y, z)$. Thus,

$$
z \frac{\partial Q}{\partial z} \cdot \frac{M_{\overline{1}}(x, y, Q)}{Q}=M(x, y, z)-x^{2} y Q
$$

so $\left(v z^{-1} \partial z / \partial v\right) M_{\overline{1}}(x, y, z)+x^{2} y z=M_{\mathcal{N}}(x, y, v)$ follows on replacing $z$ by $v$ and $Q$ by $z$, and, under these substitutions, the above functional equation for $Q$ becomes $v=z /(1+x z)^{2}$. The explicit expression for $M_{\overline{1}}(x, y, z)$ in terms of $M_{\mathscr{N}}(x, y, z)$ is

$$
M_{\overline{1}}(x, y, z)=\frac{1-x z}{1+x z}\left(M_{\mathscr{N}}\left(x, y, \frac{z}{(1+x z)^{2}}\right)-x^{2} y z\right) .
$$

A form of this construction has been observed earlier by Bender and Canfield [2].

3.3.2. Suppression of Bivalent Vertices. Every smooth map with the exception of a rooted cycle can be constructed uniquely from a Feynman map by edge subdivision (by vertices) as follows. Subdivide each nonroot edge. Let $\mathscr{E}$ denote the set of all subdivisions of an edge. Then the corresponding set of maps under edge subdivision 
is $\mathscr{M}_{\overline{1} \overline{2}} \circ \mathscr{E}$. Subdivide the root edge and then distinguish an edge of the subdivision. This corresponds to $\hat{\mathbf{e}} \partial \mathscr{E} / \partial \hat{\mathbf{e}}$. But a cycle can be constructed uniquely by edge subdivision of a loop $l$, and the resulting map has a unique rooting. Thus

$$
\left(\frac{\hat{\mathbf{e}} \partial}{\partial \hat{\mathbf{e}}} \mathscr{E}\right) \times\left(\mathscr{M}_{\overline{1} \overline{2}} \circ \mathscr{E}\right) \stackrel{\sim}{\rightarrow} \mathscr{M}_{\overline{1}}-\{l\} \circ \mathscr{E} .
$$

This is weight-preserving. The subdivision is accomplished by the substitution $z \mapsto$ $z(1-x z)^{-1}$. Then, since the generating series for $\{l\}$ is $x y^{2} z$,

$$
\left(z \frac{\partial}{\partial z} \frac{z}{1-x z}\right)\left(\left.\frac{M_{\overline{1} \overline{2}}(x, y, z)}{z}\right|_{z \mapsto z /(1-x z)}\right)=M_{\overline{1}}(x, y, z)-\left(\left.x y^{2} z\right|_{z \mapsto z /(1-x z)}\right) .
$$

The compositional inverse of $z \mapsto z(1-x z)^{-1}$ is $z \mapsto z(1+x z)^{-1}$. Applying the latter transformation with $\left.\left(z \frac{\partial}{\partial z} \frac{z}{1-x z}\right)\right|_{z \mapsto z /(1+x z)}=z(1+x z)$, we have

$$
(1+x z) M_{\overline{1} \overline{2}}(x, y, z)=\left.M_{\overline{1}}(x, y, z)\right|_{z \mapsto z /(1+x z)}-x y^{2} z .
$$

Then from this and from (46),

$$
M_{\overline{1} \overline{2}}(x, y, z)=\frac{x z}{1+x z}\left(-y^{2}+\frac{x y}{1+x z}-\frac{2 x y}{1+2 x z}\right)+\frac{M_{\mathcal{N}}(x, y, w)}{(1+x z)(1+2 x z)},
$$

since

$$
w=\left.v\right|_{z \mapsto z /(1+x z)}=z \frac{1+x z}{(1+2 x z)^{2}} .
$$

This is an explicit expression for $M_{\overline{1} \overline{2}}(x, y, z)$ in terms of $M_{\mathscr{N}}(x, y, z)$.

From Lagrange's theorem (41) it follows that (48) has a unique solution in $z \in \mathbb{Q}[x][[w]]$. Since the equation is quadratic in $z$, the latter can be determined explicitly as

$$
z=2 w /(1-4 x w+\sqrt{1-4 x w}),
$$

and uniquely since the other solution is not in $\mathbb{Q}[x][[w]]$. Thus, $1+2 x z=W^{-1}$, $1+x z=\frac{1}{2}\left(W+W^{-1}\right), z=2 w / W(1+W)$, where $W=\sqrt{1-4 x w}$. With the aid of these, $z$ can be eliminated from (47) to express $M_{\overline{1}}(x, y, z)$ in terms of $M_{\mathscr{N}}(x, y, w)$, and $x, y, w$. In the resulting equation, $w$ can be replaced by $z$ to give

$$
\frac{1+t}{2 t^{2}} M_{\overline{1} \overline{2}}\left(x, y, \frac{2 z}{t(1+t)}\right)=M_{\mathscr{N}}(x, y, z)-\frac{2 x z}{t(1+t)}\left(\frac{y^{2}}{t}-\frac{2 x y}{1+t}+2 x y\right),
$$

where $t=\sqrt{1-4 x z}$. Theorem $\mathrm{D}$ follows from Theorem $\mathrm{C}$, giving an explicit expression relating $M_{4}$ to $M_{\overline{1} \overline{2}}$.

It should be noted that Theorem D could also be obtained by transforming the integral in (3).

\section{Reformulation of the Partition Function for the ${ }^{4}$-Model}

In this approach to the $\phi^{4}$-model we consider the set of all maps on $k$ vertices, each having the same degree. The set of all such maps for $k=1,2, \ldots$ is the set of all vertex regular maps. We begin by deriving the genus series for 1-vertex maps, 
for which it is convenient to obtain the number of planted plane trees first. Note that 1-vertex maps on the sphere are duals of trees.

4.1. Specialisation to Trees and 1-Vertex Maps. We use the character formulation of the genus series for this purpose, although the integral representation would have served as well.

We rederive the result for trees from the genus series for maps as rooted maps with one face in the sphere. The correspondence is obtained by placing a vertex in the single face and joining it to the root vertex of the map so that, in anticlockwise circulation of this vertex, the root edge is encountered immediately after the new edge. The resulting tree is rooted at the new vertex, and is therefore a planted plane tree. Again certain results about the irreducible characters of the symmetric group are used, and are stated without proof. From Theorem B, the number of such trees on $n$ edges is $\left[x^{n+1} y z^{n}\right] M_{\mathcal{N}}(x, y, z)$. But if $\theta \vdash m$, then one can show that $[y] H_{\theta}(y)=(-1)^{k}(m-1) ! \delta_{\theta,\left[m-k, 1^{k}\right]} / f^{\theta}$, where $0 \leqq k \leqq m-1$. Furthermore, if $\theta=\left[m-k, 1^{k}\right]$, then $H_{\theta}(x)=(-1)^{m}(-x+k)_{m}$. In view of the coefficient operator, let $y^{2}=0$, so we are now working in the quotient ring with this relation. Thus $\log (1+c y)=c y$, so from $(10)$,

$$
\left[y z^{n}\right] R_{\mathscr{N}}(x, y, z)=\frac{1}{n ! 2 n} \sum_{k=0}^{2 n-1}(-1)^{k}(-x+k)_{2 n} \chi_{\left[2^{n}\right]}^{\left[2 n-k, 1^{k}\right]} .
$$

But if $\alpha=\left[1^{a_{1}} 2^{a_{2}} \ldots\right] \vdash m$, then $\sum_{r=0}^{m-1} \chi_{\alpha}^{\left[1^{r}, m-r\right]} t^{r}=(1+t)^{-1} \prod_{i=1}^{m}\left\{1-(-t)^{i}\right\}^{a_{i}}$. Thus

$$
\begin{aligned}
{\left[y z^{n}\right] M_{\mathcal{N}}(x, y, z) } & =\frac{(2 n) !}{2^{n} n !} \sum_{k=0}^{2 n-1}(-1)^{\left\lfloor\frac{k}{2}\right\rfloor}\left(\begin{array}{c}
n-1 \\
\left\lfloor\frac{k}{2}\right\rfloor
\end{array}\right)\left[w^{2 n}\right](1+w)^{-x+k} \\
& =\frac{(2 n) !}{2^{n} n !}\left[w^{2 n}\right] \sum_{k=0}^{n-1}(-1)^{k}\left(\begin{array}{c}
n-1 \\
k
\end{array}\right) \frac{(1+w)^{2 k}+(1+w)^{2 k+1}}{(1+w)^{x}} \\
& =(-1)^{n+1} \frac{(2 n) !}{2^{n} n !}\left[w^{n+1}\right](2+w)^{n}(1+w)^{-x}
\end{aligned}
$$

Let $c_{n+1}=\left[w^{n+1}\right](2+w)^{n}(1+w)^{-x}$, and let $C(t)=\sum_{n \geqq 0} c_{n} t^{n}$. Then by Lagrange's implicit function theorem (42) $C(t)=(2+w)^{-1}(1-t)^{-1}(1+w)^{-x}$, where $w(t)$ satisfies $w=t(2+w)$. Thus $w=2 t /(1-t)$ so $C(t)=\frac{1}{2}(1-t)^{x}(1+t)^{-x}$, whence

$$
\left[y z^{n}\right] M_{\mathcal{N}}(x, y, z)=(-1)^{n+1} \frac{(2 n) !}{2^{n} n !} c_{n+1}=(-1)^{n+1} \frac{(2 n) !}{2^{n+1} n !}\left[t^{n+1}\right]\left(\frac{1-t}{1+t}\right)^{x} .
$$

But $\log (1-t) /(1+t)=-2 t \xi(t)$, where $\xi(t) \in \mathbb{Q}[[t]]$ and $\xi(0)=1$. Thus, replacing $t$ by $-t$,

$$
\left[y z^{n}\right] M_{\mathscr{N}}(x, y, z)=\frac{(2 n) !}{2^{n+1} n !}\left[t^{n+1}\right]\left(\frac{1+t}{1-t}\right)^{x},
$$

so the number of planted plane trees on $n+1$ edges is

$$
\left[x^{n+1} y z^{n}\right] M_{\mathcal{N}}(x, y, z)=\frac{(2 n) !}{2^{n+1} n !(n+1) !}\left[t^{n+1}\right]\left(\log \frac{1+t}{1-t}\right)^{n+1}=\frac{1}{n+1}\left(\begin{array}{c}
2 n \\
n
\end{array}\right) .
$$


The number of 1-vertex maps can be obtained easily now. These have also been considered by Harer and Zagier [15]. The number $m_{n}(g)$ of 1-vertex maps of genus $g$ on $n$ edges is $m_{n}(g)=\left[x y^{n+1-2 g} z^{n}\right] M_{\mathcal{N}}(x, y, z)=\left[x^{n+1-2 g} y z^{n}\right] M_{\mathcal{N}}(x, y, z)$, by duality so, from (49),

$$
m_{n}(g)=\left[x^{n+1-2 g}\right]\left[y z^{n}\right] R_{\mathscr{N}}(x, y, z)=\frac{(2 n) !}{2^{n+1} n !}\left[x^{n+1-2 g} t^{n+1}\right]\left(\frac{1+t}{1-t}\right)^{x} .
$$

4.2. The Genus Series for Regular Maps. To prove Theorem E, it is first necessary to confirm that $P_{\alpha}(N)$ and $F_{n, k}(N)$ are polynomials in $N$. This may be done without employing explicit expansions. Let $\mathbb{Q}\left\langle X_{1}, \ldots, X_{k-1}\right\rangle$ denote the ring of Laurent polynomials in $X_{1}, \ldots, X_{k-1}$ with coefficients in $\mathbb{Q}$. It is evident from (12) that $\Psi_{k}(X, u) \in \mathbf{R}[[u]]$, where

$$
\begin{aligned}
\mathbf{R}= & \mathbb{Q}\left\langle X_{1}, \ldots, X_{k-1}\right\rangle\left[X_{k},\left(1-X_{k}\right)^{-1}\right], \\
& \prod_{i=1}^{k}\left(1-X_{i} / X_{i-1}\right)^{-1} \in \mathbb{Q}\left\langle X_{1}, \ldots, X_{k-1}\right\rangle\left[\left[X_{k}\right]\right] .
\end{aligned}
$$

Let $\alpha \vdash k$. Then, from (13), $\sum_{N \geqq 1} P_{\alpha}(N) X_{k}^{N-1} \in \mathbb{Q}\left[\left(1-X_{k}\right)^{-1}\right]\left[\left[X_{k}\right]\right]$. If $s$ is a positive integer, $\left[w^{N}\right](1-w)^{-s}=\left(\begin{array}{c}N+s-1 \\ s-1\end{array}\right) \in \mathbb{Q}[N]$. Thus $P_{\alpha}(N) \in \mathbb{Q}[N]$ so, from (14), $F_{n, k}(N) \in \mathbb{Q}[N]$. Thus $P_{\alpha}(y)$ and $F_{n, k}(y)$ are defined by formally replacing $N$ by $y$. This argument is implicit in the statement of Theorem $\mathrm{E}$.

4.2.1. Hermite Polynomials. If $\alpha \in \mathbb{N}^{k}$, then with the convention that $i_{k+1}=i_{1}$, we assert that

$$
P_{\alpha}(N)=\sum_{0 \leqq i_{1}, \ldots, i_{k} \leqq N-1} \prod_{j=1}^{k} \frac{1}{2^{i_{j}} i_{j} !} \psi_{i_{j}, i_{j+1}}\left(\alpha_{j}\right),
$$

where, for nonnegative integers $n, r, s$,

$$
\psi_{r, s}(n)=\frac{1}{\sqrt{\pi}} \int_{\mathbb{R}} x^{n} H_{r}(x) H_{s}(x) e^{-x^{2}} d x,
$$

and the Hermite polynomials $H_{n}(x)$ are defined by $H_{n}=2 x H_{n-1}-2(n-1) H_{n-2}$ for $n \geqq 2, H_{0}=1, H_{1}=2 x$. Their standardisation is $2^{n}$, and their orthogonality relation is

$$
\int_{\mathbb{R}} H_{r}(x) H_{s}(x) e^{-\frac{1}{2} x^{2}} d x=\frac{n !}{2^{2 k} k !} \sqrt{\pi} .
$$

The proof involves showing that $P_{\alpha}(N)$, defined by (13), and $Q_{\alpha}(N)$, defined by the right-hand side of (51), have the same generating series, and are therefore equal. Now

$$
Q_{\alpha}(N)=\alpha !\left[u^{\alpha}\right]\left[\left(x_{1} \cdots x_{k}\right)^{N-1}\right] \Phi_{k}\left(\frac{1}{2} \mathbf{x}, \mathbf{u}\right) \prod_{i=1}^{k}\left(1-x_{i}\right)^{-1}
$$

where

$$
\Phi_{k}(\mathbf{x}, \mathbf{u})=\sum_{\substack{i_{1}, \ldots, i_{k} \geqq 0 \\ n_{1}, \ldots, n_{k} \geqq 0}} \prod_{j=1}^{k} \psi_{i_{j}, i_{j}+1}\left(n_{j}\right) \frac{x_{j}^{i_{j}} u_{j}^{n_{j}}}{i_{j} ! n_{j} !}
$$


4.2.2. An Inverse Problem and Superposition of Matchings. We therefore proceed by considering the inverse problem of determining an explicit expression for $\Phi_{k}$, by constructing the superposition of an appropriate set of combinatorial structures. The generating function for Hermite polynomials is $\sum_{n \geqq 0} H_{n}(x) \frac{t^{n}}{n !}=e^{2 x t-t^{2}}$ so, by completing the square in $2 x t-t^{2}$, it follows from (52) that

$$
\sum_{r, s, n \geqq 0} \psi_{r, s}(n) \frac{y^{r}}{r !} \frac{z^{s}}{s !} \frac{u^{n}}{n !}=e^{\frac{1}{2} \alpha u^{2}+u y+u z+\beta y z},
$$

where $\alpha=\frac{1}{2}$ and $\beta=2$. This is rewritten as

$$
\sum_{i_{j}, i_{j+1}, n_{j} \geqq 0} \psi_{i_{j}, i_{j+1}}\left(n_{j}\right) \frac{x_{j}^{l_{j}}}{i_{j} !} \frac{x_{j+1}^{i_{j+1}}}{i_{j+1} !} \frac{u_{j}^{n_{j}}}{n_{j} !}=e^{\frac{1}{2} \alpha u_{j}^{2}+u_{j} x_{j}+u_{j} x_{j+1}+\beta x_{j} x_{j+1}}
$$

where $\alpha$ and $\beta$ are now to be indeterminates, so $\psi_{r, s}(n)$ is a polynomial in $\alpha$ and $\beta$. We now reconstruct a combinatorial configuration with this as its exponential generating series. The set of primes of the configuration has $\frac{1}{2} \alpha u_{j}^{2}+u_{j} x_{j}+u_{j} x_{j+1}+$ $\beta x_{j} x_{j+1}$ as its (exponential) generating series. These primes are constructed on three disjoint sets $\mathscr{U}_{j}, \mathscr{X}_{j}, \mathscr{X}_{j+1}$ of subobjects, the numbers of which in each prime are marked by the indeterminates $u_{j}, x_{j}, x_{j+1}$ respectively. These sets may be realised as sets of labelled vertices and, in terms of this realisation: $\frac{1}{2} \alpha u_{j}^{2}$ is the generating series for two vertices in $\mathscr{u}_{j}$ connected by an edge marked by $\alpha ; u_{j} x_{j}$ is the generating series for a vertex in $\mathscr{U}_{j}$ and a vertex in $\mathscr{X}_{j}$ connected by an edge; $u_{j} x_{j+1}$ is the generating series for a vertex in $\mathscr{U}_{j}$ and a vertex in $\mathscr{X}_{j+1}$ connected by an edge; $\beta x_{j} x_{j+1}$ is the generating series for a vertex in $\mathscr{X}_{j}$ and a vertex in $\mathscr{X}_{j+1}$ joined by an edge marked with $\beta$.

Let $\mathscr{F}_{j}$ be the set of graphs enumerated by (54). Then $\mathscr{F}_{j}$ is a set of 1 -matchings on $\mathscr{U}_{j}, \mathscr{X}_{j}, \mathscr{X}_{j+1}$, satisfying the above four conditions, and with edges having "colour" $j$. Thus $\psi_{r, s}(n)$ is the number of graphs of $\mathscr{F}_{j}$ of a particular weight specified by $(r, s, n)$. Then, by superposition, $\Phi_{k}$ is the generating series for the number $\prod_{j=1}^{k} \psi_{i j, i_{j+1}}\left(n_{j}\right)$ of graphs in $\mathscr{F}_{1} \diamond \cdots \diamond \mathscr{F}_{k}$ with a certain weight. It follows from (38) that $e^{H}$ is the generating series for $\mathscr{F}_{1} \diamond \cdots \diamond \mathscr{F}_{k}$, so $\Phi_{k}=e^{H}$, where $H$ is the generating series for the set $\mathscr{H}$ of components of $\mathscr{F}_{1} \diamond \cdots \diamond \mathscr{F}_{k}$. An explicit expression for $H$ is obtained by characterizing these components as cycles and paths with coloured edges occurring in prescribed order. The detailed case analysis of $\mathscr{H}$ is an extension of the combinatorial observations in (39). We conclude that $\Phi_{k}\left(\frac{1}{2} \mathbf{x}, \mathbf{u}\right)=\Psi_{k}(X, \mathbf{u})$, from (12), so (51) follows by replacing $x_{j}$ by $X_{j} / X_{j-1}$.

4.2.3. The Genus Series for Regular Maps. If $\mathbf{M}$ is a square matrix whose elements are formal power series in $x$ with zero as their constant terms, then $\operatorname{det} \mathbf{M}$, $\operatorname{det}(\mathbf{I}+\mathbf{M})$, and $\log (\mathbf{I}+\mathbf{M})$ are defined. It follows from Jacobi's theorem for the ring of such matrices (see, for example, [11], para. 1.1.10.5) that if $d_{0}=d_{\theta_{1}} d_{\theta_{2}} \ldots$ and $\phi_{i, j}$ is a function defined on the nonnegative integers, then

$$
\log \left\{1+\sum_{\theta} d_{\theta} S_{\theta} x^{|\theta|}\right\}=\sum_{\theta}(-1)^{l(\theta)-1} \frac{1}{l(\theta)} d_{\theta} R_{\theta} x^{|\theta|},
$$


where both sums are over partitions, and

$$
\begin{aligned}
& S_{\theta}=\frac{1}{m(\theta) !} \sum_{\sigma \in \mathfrak{S}_{l(\theta)} \operatorname{sgn}(\sigma)} \sum_{0 \leqq i_{1}, \ldots, i_{l(\theta)} \leqq N-1} \prod_{r=1}^{l(\theta)} \phi_{i_{r}, i_{\sigma(r)}}\left(\theta_{r}\right), \\
& R_{\theta}=\frac{1}{m(\theta) !} \sum_{\sigma \in \mathfrak{S}_{l(\theta)} 0 \leqq i_{1}, \ldots, i_{l(\theta)} \leqq N-1} \prod_{r=1}^{l(\theta)} \phi_{i_{r}, i_{r+1}}\left(\theta_{\sigma(r)}\right) .
\end{aligned}
$$

In view of (3) and (8), properties of $\mathscr{J}^{(N)}(f)=\int_{\mathbb{R}^{N}} f(\lambda) V^{2}(\lambda) e^{-p_{2}} d \lambda$ are required, where $f \in \mathbb{C}[[\lambda]]$. Let $n, k, k_{1}, \ldots, k_{l}$ be positive integers. Then

$$
\begin{aligned}
\mathscr{J}^{(N)}(1) & =\sqrt{\pi}^{N} 1 ! \cdots N ! / \sqrt{2}^{N(N-1)}, \\
\mathscr{J}^{(N)}\left(p_{n}^{k}\right) & =\sum_{\theta \vdash k} \frac{k !}{\theta ! m(\theta) !}(N)_{l} \mathscr{J}^{(N)}\left(\lambda_{1}^{n \theta_{1}} \cdots \lambda_{l}^{n \theta_{l}}\right), \\
\mathscr{J}^{(N)}\left(\lambda_{1}^{k_{1}} \cdots \lambda_{l}^{k_{l}}\right) & =\frac{\mathscr{J}^{(N)}(1)}{(N)_{l}} \sum_{0 \leqq \beta_{1}, \ldots, \beta_{l} \leqq N-1} \sum_{\pi \in \mathfrak{S}_{l}} \operatorname{sgn}(\pi) \prod_{j=1}^{l} \frac{\psi_{\beta_{j}, \beta_{\pi(j)}}\left(k_{j}\right)}{2^{\beta_{j}} \beta_{j} !} .
\end{aligned}
$$

Equation (56) is well known, and is related to the volume of the unitary group. It is an immediate consequence of the orthogonality relation (53) for Hermite polynomials, and the invariance of the determinant of the Vandermonde matrix under replacement of its $(i, j)$-element $x_{i}^{j-1}$ by a monic polynomial of degree $j-1$ in $x_{i}$.

With these preliminaries, we may now prove Theorem $\mathrm{E}$ by establishing (14). From the integral representation (3) and (8) for the genus series for maps, the genus series for regular maps is $W(z, y)$, where $W(z, N)=2 z \frac{\partial}{\partial z} \sum_{n \geqq 1} A_{n / 2}$ and

$$
A_{n}=\log \left\{\frac{1}{\sqrt{2 \pi}^{N} \prod_{j=1}^{N} j !} \int_{\mathbb{R}^{N}} V^{2}(\lambda) e^{-\frac{1}{2} p_{2}+\frac{1}{2 n} z^{n} x p_{2 n}} d \lambda\right\} .
$$

But $\int_{\mathbb{R}^{N}} V^{2}(\lambda) e^{-\frac{1}{2} p_{2}} d \lambda=\sqrt{2 \pi}^{N} \prod_{j=1}^{N} j$ !, from (56). Then

$$
A_{n}=\log \left\{1+\sum_{j \geqq 1} \frac{G_{j}(n, y)}{2^{j} j ! n^{j} 2^{n j}} z^{n j} x^{j}\right\}
$$

where

$$
G_{k}(n, N)=\frac{1}{\sqrt{2 \pi}^{N} \prod_{j=1}^{N} j !} \int_{\mathbb{R}^{N}} V^{2}(\lambda) e^{-\frac{1}{2} p_{2}} p_{2 n}^{k} d \lambda
$$

Then, from (57) and (58),

$$
G_{k}(n, N)=2^{k n} \sum_{\theta \vdash k} \frac{k !}{m(\theta) ! \theta !} \sum_{\beta \in \Xi_{l(\theta)}} \operatorname{sgn}(\beta) \sum_{0 \leqq i_{1}, \ldots, i_{l(\theta)} \leqq N-1} \prod_{j=1}^{l(\theta)} \frac{1}{2^{i_{J}} i_{j} !} \psi_{i_{j}, i_{\beta(j)}}\left(2 n \theta_{j}\right) .
$$

Thus, from (59) and (55),

$$
A_{n}=\sum_{\theta}(-1)^{l(\theta)-1} \frac{1}{l(\theta)} \frac{1}{\theta ! m(\theta) !}\left(\frac{x}{2 n}(2 z)^{n}\right)^{|\theta|} l(\theta) ! P_{2 n \theta}(N)
$$

and the result follows. Thus the proof of Theorem $\mathrm{E}$ is complete. 
4.3. Basis Polynomials for the Genus Series. Theorem E expresses the genus series for regular maps as a linear combination of the $P_{\alpha}(y)$, and they therefore form a basis set of polynomials of the genus series for the special cases of regular maps. We now consider the consequences of the presentation of the genus series for 1-vertex maps and 2-vertex maps with respect to this basis. It is known from the Euler-Poincare formula that, when the number of edges is fixed, the genus series for regular maps with $k$ vertices has a prescribed parity in $y$. There are two possible difficulties.

4.3.1. Parity-Respecting Forms. The first is that the presentation of $P_{\alpha}(y)$ obtained from Theorem $\mathrm{E}$ does not clearly exhibit this parity. The genus series for 1-vertex maps is an example of this. From Theorem E, the genus series for 1-vertex maps on $n$ edges is $2^{n} P_{[2 n]}(y)$. There is a minor abuse of terminology in the case of regular maps in referring to this series as genus series since the indeterminate $y$ marks faces, not genus. However, for graphs on $n$ edges and one vertex, the genus is immediately determined by the Euler-Poincare formula. The number $m_{g}(n)$ of 1-vertex maps of genus $g$ on $n$ edges is $(2 n) ! / 2^{n} n$ ! times the coefficient of $y^{n+1-2 g}$ in

$$
\sum_{k=1}^{n+1}\left(\begin{array}{l}
y \\
k
\end{array}\right)\left(\begin{array}{c}
n \\
k-1
\end{array}\right) 2^{k-1}
$$

since such maps have $n+1-2 g$ faces. This agrees with (50). Although from topological considerations, the series has parity $n+1 \bmod 2$, and this is not evident from its presentation. When $n=2 m$ we transform this polynomial into

$$
\sum_{j=0}^{m} 4^{j}\left(\begin{array}{c}
m \\
j
\end{array}\right) \frac{(y-j)^{(2 j+1)}}{(2 j+1) !}
$$

a parity-respecting form of the genus series for 1-vertex maps, since each polynomial in the basis $\mathbf{B}=\left\{(y-j)^{(2 j+1)}: j \geqq 0\right\}$ has odd parity. This basis is well adapted to hypergeometric analysis. The odd case can be treated in a similar way.

The same difficulty is exhibited by the genus series for regular maps with $k$ vertices since the $P_{\alpha}(y)$ individually do not have the correct parity for a genus series. The genus series for 2-vertex maps, maps with precisely two vertices, these being of the same degree, is an example of this. The genus series for 2-vertex maps on $2 n$ edges is $\frac{4^{n}}{2 n}\left\{P_{[4 n]}(y)-P_{\left[(2 n)^{2}\right]}(y)\right\}$. It can be checked that neither $P_{[4 n]}(y)$ nor $P_{\left[(2 n)^{2}\right]}(y)$ have a parity. Since their difference is the genus series (up to a factor) for 2-vertex maps, terms of the opposite parity cancel when the series is reexpressed with respect to the basis $\left\{1, y, y^{2}, \ldots\right\}$ of monomials in $y$. We conclude that, in general, the $P_{\alpha}(y)$ do not serve as a parity-respecting basis for the genus series for regular maps.

However, as with 1-vertex maps, there is a parity-respecting presentation of this series. With the use of properties of symmetric functions, if follows that the number of 2-vertex maps $d_{g}(n)$ of genus $g$ with $n$ edges is $(n-1)$ ! times the coefficient of $y^{n-2 g}$ in

$$
\sum_{j=0}^{\left\lfloor\frac{1}{2}(n-1)\right\rfloor}\left(\begin{array}{c}
2 j \\
j
\end{array}\right) \sum_{r=0}^{n-2 j-1}\left(\begin{array}{c}
n-2 j-1 \\
r
\end{array}\right)\left(\begin{array}{c}
y+j+r \\
n
\end{array}\right) \stackrel{\left\lfloor\frac{1}{2}(n-2 j-1)\right\rfloor}{\sum_{k=0}} \frac{1}{4^{k}}\left(\begin{array}{c}
2 k \\
k
\end{array}\right)\left(\begin{array}{c}
n \\
2 k
\end{array}\right)
$$


since such maps have $n-2 g$ faces. This presentation of this series does not exhibit the parity that the series possesses. However, this can be resolved by expressing the series with respect to the basis $\mathbf{B}$. With the use of hypergeometric analysis it can be shown [1] that the number of 2-vertex maps of genus $g$ on $2 m$ edges is $(2 m) !\left(\begin{array}{c}2 m \\ m\end{array}\right)$ times the coefficient of $y^{2 m-2 g-1}$ in

$$
\sum_{i=0}^{m-1} \frac{4^{i-m}(y-i)^{(2 i+1)}}{(2 i+1) !(i+1)}\left(\begin{array}{c}
m-1 \\
i
\end{array}\right) \sum_{k=0}^{m-i-1} \frac{(-1)^{k}}{2 k+1}\left(\begin{array}{c}
m-i-1 \\
k
\end{array}\right)\left(\begin{array}{c}
2 m-k-1 \\
m
\end{array}\right) .
$$

This form is parity-respecting. The odd case can be treated in a similar way.

A symmetric function argument cannot be used to determine the genus series for regular maps with $k \geqq 2$ vertices since the analogous series are no longer symmetric.

4.3.2. Degree-Respecting Forms. The second difficulty concerns the degree of the genus series for regular maps with $k$ vertices. From Theorem E, the degree of $P_{\alpha}(y)$ as a polynomial in $y$ is $\frac{1}{2}|\alpha|$, and the degree of the genus series for regular maps with $k$ vertices is at most the maximum of the degrees of the polynomials $P_{\alpha}(y)$ in its support. However, the degree of the genus series may not attain the maximum because of the existence of identities among the family of basis polynomials $P_{\alpha}$. Although this does not arise in the case of 1-vertex maps, for 2-vertex maps both $P_{[4 n]}(y)$ and $P_{\left[(2 n)^{2}\right]}(y)$ have degree $n$ as polynomials in $y$. However, the terms of top degree cancel in their difference, and the degree of the 2-vertex map series is not the degree of the highest degree polynomial in the support of the series. The basis $P_{\alpha}(y)$ does not afford a degree-respecting basis for the genus series for 2-vertex maps.

Both (60) and (61) are degree-respecting presentations of the genus series for 1 -vertex maps and 2-vertex maps, so these are both parity- and degree-respecting presentations of the two series. In view of this, it is reasonable to ask whether it is possible to construct a basis which is parity-respecting and degree-respecting for all vertex regular maps, rather than for just 1-vertex maps and 2-vertex maps, as part of an attack on the $\phi^{4}$-model.

4.3.3. Topologically Good Forms. It is advantageous to have the genus series in a form that facilitates specialisation to particular surfaces. We call such bases topologically good. It may be possible to construct topologically good bases from linear combinations of the $P_{\alpha}$, although it is unclear how this can be done. For example, for the genus series for 1-vertex maps and 2-vertex maps, specialisation to the sphere is immediate. This is because this case corresponds to the coefficient of the term in $y$ of highest degree. It is readily seen that $m_{0}(n)=\frac{1}{n+1} c(n), d_{0}(2 m)=c(m)^{2}$, and $d_{0}(2 m+1)=c(m)^{2}$, where $c(n)=(2 n) ! / n !^{2}$. Specialisation to the torus is possible, since this involves the term of next highest degree in $y$, the basis $\mathbf{B}$ being graded by degree.

\section{Hypermaps}

5.1. The Embedding Theorem for Hypermaps. Let $p$ be a positive integer. Then $\sigma=\left(g_{1}, \ldots, g_{p}\right) \in \mathfrak{\Xi}_{n}^{p}$ is a permutation system for $\pi \in \Xi_{n}$ of type $\left(t_{1}, \ldots, t_{p}\right)$ and order $p$ if $\pi=g_{1} \cdots g_{p}$. A permutation system $(\rho, \sigma)$ on a finite set $\mathscr{S}$, such that the group generated by $\sigma$ and $\rho$ is transitive on $\mathscr{S}$, is called a hypermap [5]. 
A hypermap is therefore a generalisation of a rotation system since the condition that one of the permutations be a fixed point free involution no longer applies. Let $G$ be a rooted 2-face-colourable map, with edge set $E(G)=\{1, \ldots, n\}$, embedded in an orientable surface $\Sigma$. Directions are assigned to the edges of $G$ so that the edges of the boundary of each face are directed consistently with the root edge. Together, the cyclic lists of origins of edges encountered in traversing the boundary of a small disc, centred at each $v$, in a sense specified by the orientation of $\Sigma$, partitions $\{1, \ldots, n\}$ and represents a permutation $v \in \mathfrak{S}_{n}$. The edge labels encountered in traversing the boundary of each root-coloured face in a sense consistent with the orientation of $\Sigma$ partitions $\{1, \ldots, n\}$ and represents a permutation $\phi \in \mathfrak{S}_{n}$. The corresponding permutation for non-root-coloured faces of $G$ is denoted by $\varepsilon$. Then $G$ corresponds uniquely to the hypermap $(v, \varepsilon)$ and conversely, each hypermap $(v, \varepsilon)$ corresponds to a unique 2-face-coloured map, up to a permutation of the colours. Moreover, $\phi=v \varepsilon$. This bijection is the dual of Walsh's [31] that exhibited a bijection between hypermaps and edge-labelled bipartite maps.

Let $\mathscr{A}_{1}, \mathscr{A}_{2} \subseteq \mathscr{N}$. Let $P_{\mathscr{A}_{1}, \mathscr{A}_{2}}\left(x, y_{1}, y_{2}, z\right)$ be the generating series for permutation systems $(v, \varepsilon)$, of order 2 , in which $v \varepsilon$ has no cycles of length not in $\mathscr{A}_{1}, \varepsilon$ has no cycles of length not in $\mathscr{A}_{2}$, and in which $x, y_{1}, y_{2}$, and $z$ are indeterminates marking, respectively, the numbers of cycles in $v, v \varepsilon, \varepsilon \in \Im_{n}$ and $n$.

The genus series for maps is recoverable from the genus series for 2-facecoloured maps since, given a rooted map, each edge can be replaced by a digon. This operation is reversible, and does not affect the genus, so

$$
M_{\mathscr{A}}\left(x, y_{1}, z^{2} y_{2}\right)=C_{\{2\}, \mathscr{A}}\left(x, y_{1}, y_{2}, z\right)
$$

where it is recalled that $M_{\mathscr{A}}(x, y, z)$ is the genus series for rooted maps having no faces of degree not in $\mathscr{A}$.

Not every $(v, \varepsilon) \in \Xi_{n}^{2}$ corresponds to a hypermap, since $v$ and $\varepsilon$ may not generate $\mathfrak{\Xi}_{n}$. However, each such element is a permutation system for an unordered collection of hypermaps, and the cycles of $v \varepsilon$ describe the root-coloured faces of the embeddings of the unordered collection. Thus $\log P_{\mathscr{A}_{1}, \mathscr{A}_{2}}$ is the generating series for edge-labelled 2-face-coloured maps with the face restrictions specified by $\mathscr{A}_{1}, \mathscr{A}_{2}$. Thus, from the bijection, the genus series $C_{\mathscr{A}_{1}, \mathscr{A}_{2}}\left(x, y_{1}, y_{2}, z\right)$ for rooted 2 -face-coloured maps is

$$
C_{\mathscr{A}_{1}, \mathscr{A}_{2}}\left(x, y_{1}, y_{2}, z\right)=z \frac{\partial}{\partial z} \log P_{\mathscr{A}_{1}, \mathscr{A}_{2}}\left(x, y_{1}, y_{2}, z\right),
$$

where the derivative accounts for the fact that each rooted map on $n$ edges corresponds to $(n-1)$ ! edge-labelled maps. From the definition of $P_{\mathscr{A}_{1}, \mathscr{A}_{2}}$,

$$
P_{\mathscr{A}_{1}, \mathscr{A}_{2}}\left(x, y_{1}, y_{2}, z\right)=\sum_{n \geqq 0} \frac{z^{n}}{n !} \sum_{v \vdash n} \sum_{\phi \in \Pi_{\mathscr{A}_{1}}} \sum_{\varepsilon \in \Pi_{\mathscr{A}_{2}}} x^{l(v)} y_{1}^{l(\phi)} y_{2}^{l(\varepsilon)}\left|\mathscr{C}_{v} \mathscr{C}_{\varepsilon} \cap \mathscr{C}_{\phi}\right| \text {, }
$$

so, from (36) and from (28),

$$
P_{\mathscr{A}_{1}, \mathscr{A}_{2}}\left(x, y_{1}, y_{2}, z\right)=\sum_{n \geqq 0} \frac{z^{n}}{(n !)^{2}} \sum_{\substack{\phi \in \Pi_{\mathscr{A}} \\ \varepsilon \in \Pi_{\mathscr{A}_{2}}}} h^{\phi} y_{1}^{l(\phi)} h^{\varepsilon} y_{2}^{l(\varepsilon)} \sum_{\theta \vdash n} \chi_{\phi}^{\theta} \chi_{\varepsilon}^{\theta} H_{\theta}(x) .
$$


The following symmetries are evident.

$\left.\begin{array}{l}\text { 1) } C_{\mathscr{N}, \mathscr{N}}\left(x, y_{1}, y_{2}, z\right) \text { is a symmetric function in } x, y_{1}, y_{2}, \\ \text { 2) } C_{\mathscr{A}_{1}, \mathscr{N}}\left(x, y_{1}, y_{2}, z\right) \text { is a symmetric function in } x, y_{2}, \\ \text { 3) } C_{\mathscr{A}_{1}, \mathscr{A}_{2}}\left(x, y_{1}, y_{2}, z\right)=C_{\mathscr{A}_{2}, \mathscr{A}_{1}}\left(x, y_{2}, y_{1}, z\right) .\end{array}\right\}$

5.2. The Factorisation. Certain $P_{\mathscr{A}_{1}, \mathscr{A}_{2}}$ admit a factorisation into generating series for other permutation systems. The specialisations that are sufficient for present purposes state that

$$
\begin{gathered}
P_{\{p\}, \mathcal{N}}\left(x, y_{1}, y_{2}, z\right)=\sum_{n \geqq 0} \frac{z^{p n}}{(p n) !} \frac{y_{1}^{n}}{p^{n} n !} \sum_{\theta \vdash p n} f^{\theta} \chi_{\left[p^{n}\right]}^{0} H_{\theta}(x) H_{\theta}\left(y_{2}\right), \\
P_{\{p\},\{p r\}}\left(x, y_{1}, y_{2}, z\right)=\sum_{n \geqq 0} \frac{z^{p r n} y_{1}^{r n} y_{2}^{n}}{p^{r n}(r n) !(r p)^{n} n !} \sum_{\theta \vdash p r n} \chi_{\left[(p r)^{n}\right]}^{\theta} \chi_{\left[p^{r n}\right]}^{0} H_{\theta}(x) .
\end{gathered}
$$

By applying the bijection $\Delta_{p}$ defined in (30) and by rearranging the sum we have, from (33)

$$
\begin{aligned}
P_{\{p\},\{p r\}}\left(x, y_{1}, y_{2}, z\right)= & \sum_{n \geqq 0} \frac{z^{p r n} y_{1}^{r n} y_{2}^{n}}{p^{r n}(r n) !(r p)^{n} n !} \sum_{\substack{s_{1}, \ldots, s_{p} \geqq 0 \\
s_{1}+\cdots+s_{p}=n}}\left(\begin{array}{c}
n \\
s_{1}, \ldots, s_{p}
\end{array}\right)\left(\begin{array}{c}
r n \\
r s_{1}, \ldots, r s_{p}
\end{array}\right) \\
& \cdot \sum \prod_{j=1}^{p} f^{\theta^{(J)}} \chi_{\left[r^{s_{j}}\right]}^{\theta^{(j)}} H_{p}^{\theta^{(j)}}(x-j+1),
\end{aligned}
$$

where the third summation is over all $\left(\theta^{(1)}, \ldots, \theta^{(p)}\right)$ such that $\theta^{(j)} \vdash r s_{j}$ for $j=1, \ldots, p$, and where $H_{p}^{\theta}(x)$ is defined in (31). Thus

$$
P_{\{p\},\{p r\}}\left(x, y_{1}, y_{2}, z\right)=\bar{A}\left(x, y_{1}, y_{2}, z\right) \cdots \bar{A}\left(x-p+1, y_{1}, y_{2}, z\right),
$$

where

$$
\bar{A}\left(x, y_{1}, y_{2}, z\right)=\sum_{\substack{\theta \\|\theta| \equiv 0 \bmod r}} \frac{z^{p|\theta|} y_{1}^{|\theta|} y_{2}^{\frac{|\theta|}{r}}}{p^{|\theta|}\left(\frac{|\theta|}{r}\right) !(r p)^{\frac{|\theta|}{r}}|\theta| !} f^{\theta} \chi_{\left[r^{|\theta| / r}\right]}^{\theta} H_{p}^{\theta}(x) .
$$

Then, by rearrangement of the series, we obtain the desired factorisation

$$
\begin{aligned}
\bar{A}\left(x, y_{1}, y_{2}, z\right)= & \sum_{n \geqq 0} \frac{\left(p^{p r-r-1} z^{p r} y_{1}^{r} y_{2}\right)^{n}}{n ! r^{n}(r n) !} \\
& \times \sum_{\theta \vdash r n} f^{\theta} \chi_{\left[r^{n}\right]}^{\theta} H_{\theta}\left(\frac{x}{p}\right) H_{\theta}\left(\frac{x+1}{p}\right) \cdots H_{\theta}\left(\frac{x+p-1}{p}\right) .
\end{aligned}
$$

We now derive a relationship between $2 r$-regular maps and a class of 2-facecoloured maps (those whose root-coloured faces have degree $r$ ). From (65) with $p=2$, we have, $\bar{A}\left(x, y_{1}, y_{2}, z\right)=P_{\{r\}, N}\left(\frac{1}{2} x, \frac{1}{2} y_{2}, \frac{1}{2}(x+1), 2 z^{2} y_{1}\right)$, so from (66)

$$
P_{\{2\},\{2 r\}}\left(x, y_{1}, y_{2}, z\right)=U(x) U(x-1),
$$


where $U(x)=P_{\{r\}, \mathscr{N}}\left(\frac{1}{2} x, \frac{1}{2} y_{2}, \frac{1}{2}(x+1), 2 z^{2} y_{1}\right)$. Then, from (63)

$$
\begin{aligned}
& C_{\{2\},\{2 r\}}\left(x, y_{1}, y_{2}, z\right) \\
& =z \frac{\partial}{\partial z} \log P_{\{r\}, N}\left(\frac{x}{2}, \frac{y_{2}}{2}, \frac{x+1}{2}, 2 z^{2} y_{1}\right) P_{\{r\}, \mathcal{N}}\left(\frac{x-1}{2}, \frac{y_{2}}{2}, \frac{x}{2}, 2 z^{2} y_{1}\right)
\end{aligned}
$$

from (67). But $\left(z / 2 z^{2} y_{1}\right) \partial\left(2 z^{2} y_{1}\right) / \partial z=2$, so from (63) and (62),

$$
\begin{aligned}
& \frac{1}{2} M_{\{2 r\}}\left(x, y_{1}, y_{2} z^{2}\right) \\
& =C_{\{r\}, \mathscr{N}}\left(\frac{x}{2}, \frac{y_{2}}{2}, \frac{x+1}{2}, 2 z^{2} y_{1}\right)+C_{\{r\}, \mathcal{N}}\left(\frac{x-1}{2}, \frac{y_{2}}{2}, \frac{x}{2}, 2 z^{2} y_{1}\right) \\
& =C_{\{r\}, \mathscr{N}}\left(\frac{x+1}{2}, \frac{y_{2}}{2}, \frac{x}{2}, 2 z^{2} y_{1}\right)+C_{\{r\}, \mathscr{N}}\left(\frac{x-1}{2}, \frac{y_{2}}{2}, \frac{x}{2}, 2 z^{2} y_{1}\right)
\end{aligned}
$$

from (64). Now replace $y_{1}, y_{2}$ by $y$, and then replace $z^{2} y$ by $z$, to obtain Theorem F. This establishes the relationship between the two classes of maps.

The relationship given in Theorem $\mathrm{C}$ associated with rooted quadrangulations of arbitrary genus is recovered by setting $r=2$ in Theorem $\mathrm{F}$ and using (62). By putting $p=3$ in (65) and repeating the above arguments, one can obtain a relationship between 2-face-coloured triangulations on $3 n$ edges and all 2-face-coloured maps on $n$ edges.

Acknowledgements. One of us (DMJ) thanks G.E. Andrews, P. Ginsparg, C. Itzykson, and C. Vafa for useful discussions. This research was supported by grants, individually to DMJ and TIV, from the Natural Sciences and Engineering Research Council of Canada.

\section{References}

1. Andrews, G.E., Jackson, D.M., Visentin, T.I.: A hypergeometric analysis of the genus series for a class of 2-cell embeddings in orientable surfaces. SIAM J. Mathematical Analysis, 25, 243-255 (1994)

2. Bender, E.A., Canfield, E.R.: The asymptotic number of rooted maps on a surface. J. Combinatorial Theory A 43, 244-257 (1986)

3. Bessis, D., Itzykson, C., Zuber, J.B.: Quantum field theory techniques in graphical enumeration. Adv. Applied Math. 1, 109-157 (1980)

4. Brézin, E., Kazakov, V.A.: Exactly solved field theories and closed strings. Phys. Lett. B 236, 144-150 (1990)

5. Cori, R.: Un code pour les graphes plainaires et ses applications. Thèse de Doctorat, Paris, 1973

6. Di Francesco, P., Itzykson, C.: A generating function for fatgraphs. Preprint

7. Distler, J., Vafa, C.: A critical matrix model at $c=1$. Mod. Phys. Lett. A 6, 259-270 (1991)

8. Douglas, M.R., Shenker, S.H.: Strings in less that one dimension. Nucl. Phys. 335, 635-654 (1990)

9. Dyson, F.J.: Statistical theory of energy levels of complex systems, I, II and III. J. Math. Phys. 3, 140-156, 157-165, 166-175 (1962)

10. Ginsparg, P.: Matrix models for $2 \mathrm{~d}$ gravity. Lectures given at the Trieste Summer School, July 22-25, 1991, LA-UR-91-40101 \& hepth@xxx/9112013, Los Alamos National Laboratory Report, Los Alamos NM 87545

11. Goulden, I.P., Jackson, D.M.: Combinatorial Enumeration. New York: Wiley Interscience, 1983 
12. Gross, D.J., Migdal, A.A.: A nonperturbative treatment of two dimensional quantum gravity. Nucl. Phys. B 340, 333-365 (1990)

13. Gross, D.J., Migdal, A.A.: A nonperturbative two dimensional quantum gravity. Phys. Rev. Lett. 64, 127-130 (1990)

14. Gross, J.L., Alpert, S.R.: The topological theory of current graphs. J. Combinatorial Theory B 17, 218-233 (1974)

15. Harer, J., Zagier, D.: The Euler characteristic of the moduli space of curves. Invent. Math. 85, $457-485$ (1986)

16. Heffter, L.: Ueber das Problem der Nachbargebiete. Math. Ann. 38, 477-508 (1891)

17. Hoffman, P., Richter, B.: Embedding graphs in surfaces. J. Combinatorial Theory B 36, 65-84 (1984)

18. Hooft, G.'T.: A planar diagram theory for string interactions. Nucl. Phys. B 72, 461-473 (1974)

19. Itzykson, C., Drouffe, J-M.: Statistical Field Theory. Vol. 2, Cambridge: Cambridge University Press, 1990

20. Jackson, D.M.: On an integral representation for the genus series for 2-cell embeddings. Trans. Am. Math. Soc., 344, 755-772 (1994)

21. Jackson, D.M.: The genus series for maps. J. Pure and Applied Algebra 105, 293-297 (1995)

22. Jackson, D.M., Visentin, T.I.: A character theoretic approach to embeddings of rooted maps in an orientable surface of given genus. Trans. Am. Math. Soc. 322, 343-363 (1990)

23. Jackson, D.M., Visentin, T.I.: Character theory and rooted maps in an orientable surface of given genus: Face-coloured maps. Trans. Am. Math. Soc. 322, 365-376 (1990)

24. Jackson, D.M., Visentin, T.I.: A formulation for the genus series for regular maps. J. Combinatorial Theory A (to appear)

25. Macdonald, I.G.: Symmetric functions and Hall polynomials. Oxford: Clarendon Press, 1979

26. Mehta, M.L.: Random Matrices. London: Academic Press, 1991

27. Nelson, D., Piran, T., Weinberg, S. (eds.): Statistical Mechanics of Membranes and Surfaces. Singapore: World Scientific, 1989

28. Penner, R.C.: Perturbative series and the moduli space of Riemann surfaces. J. Differ. Geom. 27, 35-53 (1988)

29. Periwal, V., Shevitz, D.: Exactly solvable unitary matrix models: Multicritical potentials and correlates. Nucl. Phys. B 344, 731-746 (1990)

30. Serre, J-P.: Linear Representations of Finite Groups. Berlin, Heidelberg, New York: Springer, 1977

31. Walsh, T.R.S.: Hypermaps versus bipartite maps. J. Combinatorial Theory B 18, 155-163 (1975)

32. Weyl, H.: The Classical Groups. Princeton, NJ: Princeton University Press, 2nd. ed. 1946

33. Wigner, E.P.: Characteristic vectors of bordered matrices with infinite dimensions, I and II. Ann. Math. 62, 548-564 (1955) and 65, 203-207 (1957) 
